\title{
Unambiguous phase retrieval as a cophasing sensor for phased array telescopes
}

\author{
Fabien Baron, ${ }^{1,3, *}$ Isabelle Mocœur, ${ }^{1,2}$ Frédéric Cassaing, ${ }^{1}$ and Laurent M. Mugnier ${ }^{1}$ \\ ${ }^{1}$ Office National d'Etudes et de Recherches Aérospatiales, Optics Department, BP 72, 92322 Châtillon cedex, France \\ ${ }^{2}$ Centre National d'Etudes Spatiales, 18 avenue Edouard Belin, 31401 Toulouse Cedex 4, France \\ ${ }^{3}$ Current address: Cavendish Laboratory, University of Cambridge, Cambridge CB3 OHE, UK \\ *Corresponding author: baron@mrao.cam.ac.uk
}

Received August 10, 2007; revised January 4, 2008; accepted January 24, 2008; posted February 27, 2008 (Doc. ID 86344); published April 9, 2008

\begin{abstract}
Cophasing a multiple-aperture optical telescope (MAOT) or optical interferometer requires the knowledge of the tips/tilts and of the differential pistons on its subapertures. In this paper we demonstrate in the case of a point source object that a single focal-plane image is sufficient for MAOT cophasing. Adopting a least-square approach allows us to derive an analytic estimator of the subaperture aberrations, provided that these are small enough (typically for closed-loop operation) and that the pupil is diluted noncentrosymmetric. We then provide the validation of this estimator by simulations as well as a performance comparison with a more conventional iterative algorithm of phase retrieval. Finally, we present the experimental validation of both estimators on a laboratory test bench; our results, especially subnanometric repeatability, demonstrate that focalplane sensors are appropriate for the cophasing of phased array telescopes. (C) 2008 Optical Society of America OCIS codes: $010.7350,100.5070,110.5100,100.3190,120.3180$.
\end{abstract}

\section{INTRODUCTION}

Multiple-aperture optical telescopes (MAOTs) are made of a collection of subapertures, coherently combined in order to reach the diffraction limit of a single monolithic telescope whose aperture would cover the subapertures. MAOTs are now currently used for astronomy, to provide milliarcsecond resolution thanks to ground-based stellar interferometers with hectometric baselines [1-3]. Similar instruments are under study for space missions, particularly in the context of the search for exolife [4,5]. MAOTs can also be considered for wide-field space telescopes, when the primary is deployed after launch because it does not fit in the rocket fairing. Examples are the James Webb Space Telescope for astronomy [6] or multipleaperture arrays for Earth observation from a geostationary orbit [7].

Performance of optical instruments can be degraded by phase perturbations. This is particularly the case of MAOTs, operating at the diffraction limit with very long baselines or with subpupils that are not mechanically tied together with optical precision. Perturbations include quasi-static positioning errors or high-frequency contributors such as vibrations or propagation through the turbulent atmosphere. To increase the signal-to-noise ratio (SNR) of the collected data, it is necessary to cophase the array, i.e., to stabilize optical paths at zero optical path difference when interferometric data is recorded. As realtime correction over each subaperture can be performed with adaptive optics [8], the very specific issue raised by MAOTs is the correction of the differential piston/tip/tilt errors between the subapertures, as illustrated by Fig. 1. Thus the most critical component of an interferometer is the cophasing sensor, the goal of which is to measure those modes.
Many correction devices can be considered, such as tip/ tilt mirrors and delay lines in each arm, or a global deformable mirror. An obvious solution is to use a dedicated tip/tilt sensor for each subaperture and a classical interferometric (pairwise) piston sensor. But the complexity of this solution quickly increases with the number of subapertures, and the local tip/tilt measurements must be linked together.

Another solution is to retrieve aberrations from focalplane intensity measurements, an old idea first introduced by Gerchberg and Saxton [9] in the electron microscopy context and then rediscovered by Gonsalves [10].

Phase retrieval estimators use a single focal-plane image of an unresolved source observed through the instrument to reconstruct the aberrated phase, coded in the pupil transmittance. Analytic expression of the phase solution can be derived in specifically crafted experiments, such as when one has images taken with and without an exponential filter in the pupil plane [11]. However, in general, one has to rely on iterative methods to estimate the aberrations that are most compatible with the known constraints in the pupil plane and the measured images. Successful approaches include gradientsearch algorithms [12], where a least-square (LS) criterion is minimized as a function of the unknown aberrations, and iterative Fourier-transform algorithms $[10,13]$, where the estimates are Fourier-transformed back and forth to ensure compatibility with the data.

Phase retrieval has been shown for a centrosymmetric pupil to lead to a theoretical ambiguity on the sign of the even part of the phase $[10,14,15]$. In practice for monolithic instruments, the obscurations can be sufficiently asymmetric to allow phase retrieval to be used. Such is the particular case of the Hubble telescope: The telescope 


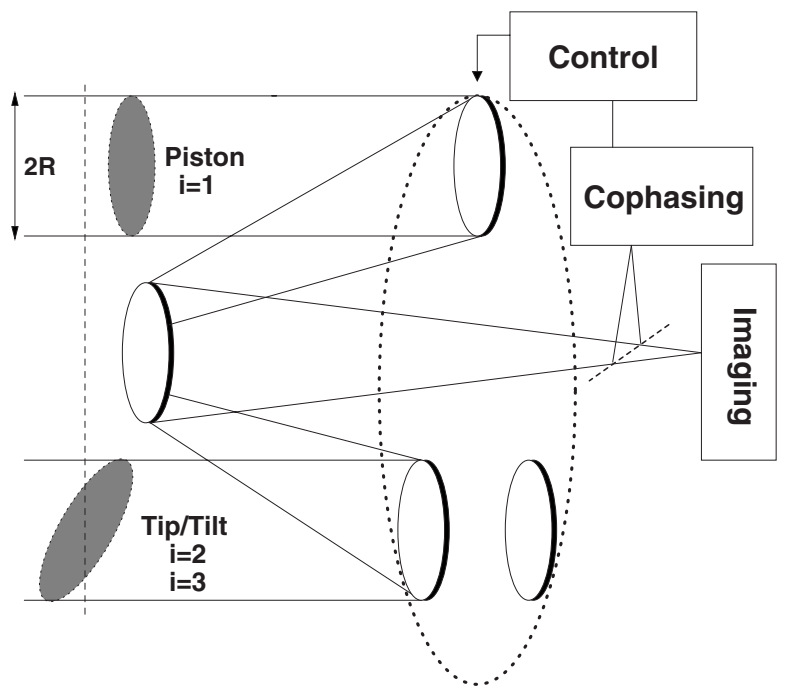

Fig. 1. Piston and tip/tilt on a MAOT.

being already in orbit, its aberrations were characterized with the blurred images of unresolved stars [16,17].

In the most general case (unknown object or any pupil configuration), it is possible to remove the sign ambiguity by using at least a second image differing by a known phase, conventionally a small defocus [18]. This phasediversity method is usually applied for the phase measurement on extended objects (see Mugnier et al. [19] for a recent review of phase diversity). Aberration measurement by phase diversity is now routinely used for the calibration of monolithic instruments [20] and has been extended to MAOTs [21-23]. While phase diversity is optically very easy to implement, it requires considerable computing power for real-time correction, as it is most often based on an iterative algorithm. Efforts in making phase diversity faster have thus been made: better numerical algorithms [24,25], modified error metric used to estimate the aberrations and object from the data [26-28], and the promising research of an analytical solution [29].

For a point source object however, one does not need the full generality of the phase diversity. The simpler phase retrieval can be used with a noncentrosymmetric aperture to retrieve the aberrated phase without ambiguity. Since MAOT pupils may be noncentrosymmetric, phase retrieval becomes an interesting solution, as the image of an unresolved source is often available. This source can be an off-axis star or an internal source and can be used for real-time cophasing or as a complementary tool for periodic calibration.

The objective of this paper is to present two focal-plane wavefront estimators, which determine simultaneously the pistons/tips/tilts on all the subapertures of a MAOT with a noncentrosymmetric pupil from the sole focalplane image of an unresolved source. In Section 2, the image formation process is described in Fourier space, leading to the derivation of the aberrated optical transfer function (OTF). In Section 3 a LS analysis is adopted for its good statistical properties [30], and a first conventional estimator is recalled, based on an iterative algorithm. In the case of a diluted nonredundant pupil and when subaperture aberrations are small, a second estima- tor requiring only a single Fourier transform-and thus particularly suited for real time cophasing-is derived. The theoretical performance of both estimators is then analyzed by simulations in Section 4. Finally, an experimental validation on the ONERA laboratory test bench BRISE allows us to compare in Section 5 the algorithms on experimental data for the DarWin [31] AstRonomical Fringe sensor (DWARF) validation.

\section{EFFECT OF ABERRATIONS ON THE IMAGE OF AN UNRESOLVED SOURCE}

\section{A. Direct Model}

The following model assumes monochromatic light and point source objects, although most of the calculations could be extended to known or centrosymmetric objects and to wide wavebands. The image $\boldsymbol{i}$ recorded by a detector is then simply the sampled point-spread function (PSF) corrupted by noise.

To simplify notation, the MAOT pupil is assumed to be formed by $N_{T}$ circular subapertures of identical diameters (Fig. 1). To implement a phase-retrieval algorithm the pupil has to be sampled, and the coordinate choice we make is such that the PSF computed from the sampled pupil matches the experimental PSF. This fixes the scaling factor of the pupil and in practice the radius $R$ (in pixels) of each subaperture.

Each subaperture $n$ is also characterized by the coordinate of its center $\boldsymbol{u}_{n}$ and by its complex transmission $\boldsymbol{p}_{n}$. The total pupil transmission can then be written as

$$
\boldsymbol{p}=\sum_{n=1}^{N_{T}} \boldsymbol{p}_{n} \star \delta_{\boldsymbol{u}_{n}},
$$

where $\delta_{\boldsymbol{u}_{n}}$ denotes a shift by the vector $\boldsymbol{u}_{n}$.

An extensive treatment of the problem would require the use of a different transmission function for each subaperture, which would allow us to take the obscurations (secondary mirrors, spiders) into account. However, for clarity of exposition, all obscurations will be neglected in the following and all transmissions will be assumed equal to unity. The modulus of the subaperture transmission $\boldsymbol{p}_{n}$ is then described by the disk function $\mathbf{\Pi}$ :

$$
\Pi(\boldsymbol{u})=\left\{\begin{array}{ll}
1 & \text { for } 0 \leq|\boldsymbol{u}| \leq R \\
0 & \text { elsewhere }
\end{array} .\right.
$$

The phase of $\boldsymbol{p}_{n}$ is expanded on a local basis of scaled Zernike polynomials [32] so that

$$
\boldsymbol{p}_{n}(\boldsymbol{u})=\boldsymbol{\Pi}(\boldsymbol{u}) \exp \left[j \sum_{k=1}^{k_{\max }} a_{k n} \boldsymbol{Z}_{\boldsymbol{k}}(\boldsymbol{u})\right]
$$

where $j^{2}=-1$ and $a_{k n}$ is the rms amplitude in radians of the mode of order $k$ on the subaperture $n$. As all apertures have the same radius, they share the same modes. Since the MAOT-specific aberrations are pistons $(k=1)$ and tips/ tilts $(k=\{2,3\})$, we will consider $k_{\max }=3$. 


\section{B. OTF Characteristics}

The autocorrelation $\boldsymbol{s}$ of the pupil is given by

$$
\boldsymbol{s}(\boldsymbol{u})=(\boldsymbol{p} \otimes \boldsymbol{p})(\boldsymbol{u})=\sum_{\boldsymbol{u}^{\prime} \in \mathbb{R}^{2}} \boldsymbol{p}\left(\boldsymbol{u}^{\prime}\right) \boldsymbol{p}^{*}\left(\boldsymbol{u}+\boldsymbol{u}^{\prime}\right),
$$

and $\boldsymbol{s}$ will be called for convenience the OTF, although it is not normalized and even though the considered optical system does not need to be shift invariant. If we express explicitly the OTF as a function of the subaperture transmissions, we get

$$
\begin{aligned}
\boldsymbol{s} & =\sum_{n=1}^{N_{T}} \sum_{n^{\prime}=1}^{N_{T}}\left(\boldsymbol{p}_{n} \star \delta_{\boldsymbol{u}_{n}}\right) \otimes\left(\boldsymbol{p}_{n^{\prime}} \star \delta_{\boldsymbol{u}_{n^{\prime}}}\right) \\
& =\sum_{n=1}^{N_{T}} \sum_{n^{\prime}=1}^{N_{T}}\left(\boldsymbol{p}_{n} \otimes \boldsymbol{p}_{n^{\prime}}\right) \star \delta_{\boldsymbol{u}_{n}-\boldsymbol{u}_{n^{\prime}}} .
\end{aligned}
$$

Equation (5) demonstrates that the OTF is composed of a central peak (the zero-frequency peak) surrounded by $N_{T}\left(N_{T}-1\right)$ satellite peaks. Each pair of subapertures defines one baseline, to which correspond two symmetric peaks in the OTF. Figure 2 (top row) shows, for example, the real OTF obtained from a pupil in an equilateraltriangle layout and without aberrations. In presence of aberrations, the peaks are complex, but as the PSF is real, the OTF has Hermitian symmetry.

From Eq. (5) and Eq. (3) it is clear that the OTF is nonlinear with respect to the $\left\{a_{k n}\right\}$ coefficients, which explains the difficulty of the phase-retrieval problem.

Two aberration estimators will be derived in the following. For one of these, one of the main assumptions will be that all considered phases are small compared with $2 \pi \mathrm{rad}$. This will allow us to simplify this nonlinear problem. The small phase assumption is a realistic case for a cophasing sensor mostly used in closed loop. However, additional procedures may first be required to lock the system, i.e., to achieve a nearly cophased state (see the related discussion in Subsection 5.D).

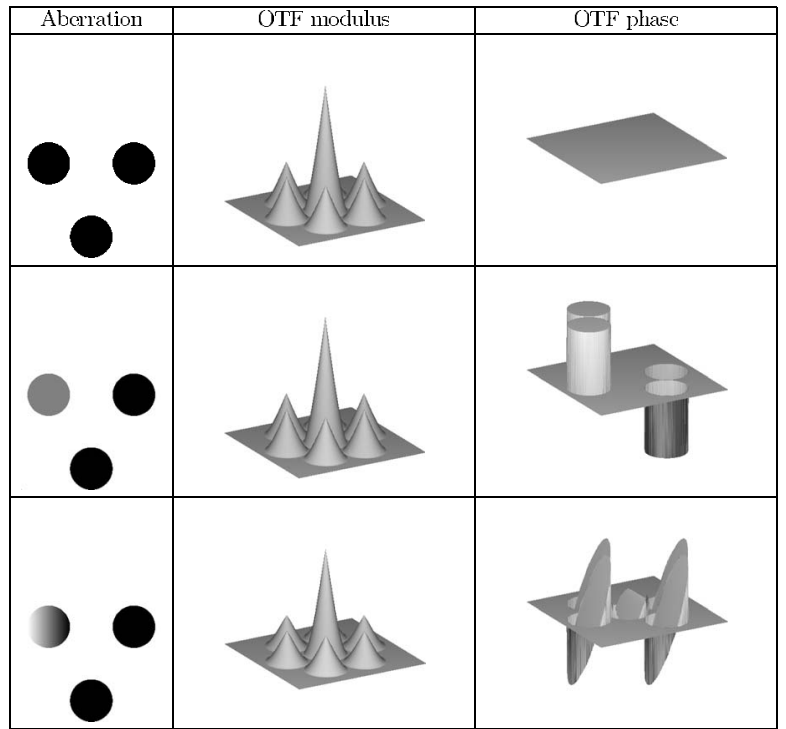

Fig. 2. Shapes of the OTF modulus and phase for no aberration (first row), a piston (second row), a tilt (third row) on one aperture.
Under the assumption of small phases, it is demonstrated in Appendix A that

$$
\boldsymbol{s} \approx \sum_{n=1}^{N_{T}} \sum_{n^{\prime}=1}^{N_{T}} \boldsymbol{\Lambda} \exp \left(j \sum_{k=1}^{3} \alpha_{n n^{\prime}}^{k} \mathcal{Z}_{k}\right) \star \delta_{\boldsymbol{u}_{n}-\boldsymbol{u}_{n^{\prime}}},
$$

where $\boldsymbol{\Lambda} \triangleq \boldsymbol{\Pi} \otimes \boldsymbol{\Pi}$ is the autocorrelation of the subaperture, which describes an OTF peak without aberration, and where by definition

$$
\alpha_{n n^{\prime}}^{k} \triangleq a_{k n}+\epsilon_{k} a_{k n^{\prime}},
$$

$$
\mathcal{Z}_{k}(\boldsymbol{u}) \triangleq \frac{1}{2} \iint_{\boldsymbol{u}^{\prime} \in \mathcal{S}(\boldsymbol{u})}\left\{\boldsymbol{Z}_{k}\left(\boldsymbol{u}^{\prime}\right)-\epsilon_{k} \boldsymbol{Z}_{k}\left(\boldsymbol{u}^{\prime}+\boldsymbol{u}\right)\right\} \mathrm{d} \boldsymbol{u}^{\prime},
$$

where $\mathcal{S}(\boldsymbol{u})$ is the overlapping area between a subaperture and itself translated by $\boldsymbol{u}$, and with $\epsilon_{k}=1$ if the radial order of $\boldsymbol{Z}_{k}$ is odd, and $\epsilon_{k}=-1$ if it is even.

Equation (6) allows us to understand the behavior of the OTF modulus and phase. When all subaperture aberrations are null, the phase of the OTF, called the phase transfer function (PTF), is null (Fig. 2, top row). In the presence of aberrations, the OTF modulus is not affected at first order; only the PTF is.

The shape of the perturbation in the PTF is then determined by the $\mathcal{Z}_{k}$ modes. Fig. 2 (center and bottom rows) presents the effects of simple aberrations in the pupil. When a piston (Fig. 2, center) or a tilt (Fig. 2, right) are applied to a single subaperture, $2\left(N_{T}-1\right)$ satellite peaks are affected. If these peaks do not overlap (see further on for a discussion on this), the OTF modulus is strictly unaffected in the piston case and barely changes in the tilt case. In the PTF a piston aberration creates phase offsets, while a tilt aberration creates phase planes. Equation (8) confirms that the first three PTF modes, the $\mathcal{Z}_{k}$ modes, are similar to Zernike modes, $\boldsymbol{Z}_{k}$, but with a double support. Thus the first $\mathcal{Z}_{k}$ modes are mutually orthogonal, which means piston measurements do not interfere with tip/tilt ones.

The amplitude of the perturbation in the PTF is measured by the coefficients $\alpha_{n n^{\prime}}^{k}$. For a piston (a mode of even radial order), it is equal to the differential aberration amplitudes between the two considered subapertures. Consequently, the piston amplitudes are antisymmetric; i.e., symmetric peaks are affected by opposite amplitudes. In contrast, for a tip or tilt (modes of odd radial order), the amplitudes of the modes are symmetric. Both peaks are affected by the same amplitude, equal to the total of the subaperture aberration amplitudes.

Thus, as long as the OTF peaks do not overlap, Eq. (7) indicates that the phase of a satellite peak $n n^{\prime}$ and of its symmetric counterpart $n^{\prime} n$ is determined entirely by the aberrations on the two subapertures $n$ and $n^{\prime}$ (whose intercorrelation forms the peak). The phase of the peaks formed by the intercorrelation of the subaperture $n$ or $n^{\prime}$ with another subaperture will also depend on one of these aberration sets, but not on both. To disentangle the two intricated sets on the peak $n n^{\prime}$, a natural idea is to check whether any kind of linear combination of the $\alpha_{n n^{\prime}}^{k}$ can allow us to retrieve all the aberrations.

This kind of approach requires the OTF peaks not to overlap, and consequently we will restrict the following 
sections to pupils with nonoverlapping peaks (cases of peak overlapping will be discussed in Subsection 3.B.4).

\section{LEAST-SQUARE ESTIMATORS}

The next subsections establish the construction of two aberration estimators based on a LS approach. They allow us to retrieve all aberrations on every subaperture from one single focal image. The first estimator is a well-known [21] iterative algorithm normally based on a two-image analysis, which we will further demonstrate to work with only one image. It will also serve as a reference in simulations. The second estimator is our analytical estimator FUSCHIA (Fast Unambiguous Sensor for CopHasing Interferometric Arrays).

Both estimators use the classic LS criterion $L$, proportional to the squared difference between the discrete Fourier transform (noted by a tilde) of the image and the OTF. In the following the noise on the image will be assumed white. If it is also additive and of Gaussian distribution, then the LS approach is equivalent to a maximum-likelihood approach. Otherwise, for other types of noise, the LS criterion still allows one to minimize the distance between those quantities. The criterion is written as

$$
L(\boldsymbol{a})=\sum_{\boldsymbol{u} \in \mathcal{D}} \frac{1}{2 \sigma^{2}}|\boldsymbol{s}(\boldsymbol{a}, \boldsymbol{u})-\widetilde{\boldsymbol{i}}(\boldsymbol{u})|^{2},
$$

where $\boldsymbol{a}$ is the aberration vector and $\sigma^{2}$ is the variance of the noise on the image. The sum is computed on the support of the OTF, the frequency domain $\mathcal{D}$ (bounded by the OTF cutoff frequency).

The vector $\hat{\boldsymbol{a}}$ of the estimated aberrations contains the aberrations that are solutions of the phase-retrieval problem, defined as the aberrations that minimize $L$ :

$$
\forall(k, n) \frac{\partial L}{\partial a_{k n}}(\hat{\boldsymbol{a}})=0 .
$$

If the real and imaginary part operators are respectively noted $\mathfrak{R}$ and $\mathfrak{I}$, then the classic expression [22] of the criterion gradient with respect to the $a_{k n}$ coefficient is expressed as

$$
\frac{\partial L}{\partial a_{k n}}=\frac{1}{\sigma^{2}} \sum_{\boldsymbol{u} \in \mathcal{D}} \mathfrak{R}\left\{(\boldsymbol{s}-\widetilde{\boldsymbol{i}}) \frac{\partial \boldsymbol{s}^{*}}{\partial a_{k n}}\right\} .
$$

\section{A. Iterative Estimator}

Using Eq. (11), the criterion can be effectively minimized by a gradient-based iterative method, such as the conjugated-gradient method.

A brief overview of the procedure is given here. The initial data are the focal image, the geometry of the input pupil, and a starting point of the aberration estimates. At each iteration, the current image is computed from the point source and the current phase estimates. The algorithm modifies the aberrations so as to decrease the gradients; then these aberrations become the current phase estimates. This process is repeated until the gradient values drop below a user-defined threshold. Iterative algorithms require several (typically 10-30) iterations to con- verge to the final aberrations, each iteration representing 2 fast Fourier transforms (FFTs) to compute. This estimator has been successfully implemented, and while its performance is excellent (see Section 4) as it achieves optimal data reconstruction in the LS sense, it inconveniently requires many FFTs to do so.

\section{B. Proposed Analytical Estimator}

In this section, we will use the small-phase approximation of the OTF from Eq. (5), in order to obtain an estimator based on an analytical formulation. This estimator uses much less processing power than the iterative algorithm previously described.

\section{Gradient and LS Criterion in Small Phases}

Let $\mathcal{D}_{0}$ be the support of the OTF central peak. We will split the computation of the criterion in Eq. (9) into two parts: one computed over $\mathcal{D}_{0}$ and the other over the rest of the frequencies $\left(\mathcal{D} \backslash \mathcal{D}_{0}\right)$. The central peak contains mixed information on only the incoherent images of all subapertures, while the useful interferometric information resides within the high-frequency satellite peaks. To keep the following calculations in this paper tractable, the part of the criterion computed on the central peak will be dismissed. The new criterion $L^{\prime}$ is then

$$
L^{\prime}(\boldsymbol{a})=\sum_{\boldsymbol{u} \in \mathcal{D} \backslash \mathcal{D}_{\mathbf{0}}} \frac{1}{2 \sigma^{2}}|\boldsymbol{s}(\boldsymbol{a}, \boldsymbol{u})-\widetilde{\boldsymbol{i}}(\boldsymbol{u})|^{2} .
$$

Note that the minimization of $L^{\prime}$ is sufficient to derive all useful information for MAOT cophasing, i.e., information about the individual aberrations on the subapertures. The gradient to minimize with respect to $a_{k n}$ is

$$
\frac{\partial L^{\prime}}{\partial a_{k n}}=\frac{1}{\sigma^{2}} \sum_{\boldsymbol{u} \in \mathcal{D} \backslash \mathcal{D}_{\mathbf{0}}} \mathfrak{R}\left\{(\boldsymbol{s}-\tilde{\boldsymbol{i}}) \frac{\partial \boldsymbol{s}^{*}}{\partial a_{k n}}\right\} .
$$

Equation (13) shows that the gradient of the criterion depends on the gradient of the conjugated OTF. The frequencies at which the OTF is constant with respect to $a_{k n}$ do not contribute to the gradient of the criterion. Thus to compute the right-hand term of Eq. (13) we need only express $\boldsymbol{s}_{k n}$, defined as the part of the OTF that depends on this coefficient $a_{k n}$. As Eq. (6) demonstrates, $\boldsymbol{s}_{k n}$ is a sum of $\left(N_{T}-1\right)$ pairs of peaks:

$$
\begin{aligned}
\boldsymbol{s}_{k n}= & \sum_{\substack{n^{\prime}=1 \\
n^{\prime} \neq n}}^{N_{T}}\left\{\left[\boldsymbol{\Lambda} \exp \left(j \sum_{k^{\prime}=1}^{3} \alpha_{n n^{\prime}}^{k^{\prime}}, \mathcal{Z}_{k^{\prime}}\right)\right] \star \delta_{\boldsymbol{u}_{n}-u_{n^{\prime}}}\right. \\
& \left.+\left[\boldsymbol{\Lambda} \exp \left(j \sum_{k^{\prime \prime}=1}^{3} \alpha_{n^{\prime} n}^{k^{\prime \prime}} \mathcal{Z}_{k^{\prime \prime}}\right)\right] \star \delta_{\boldsymbol{u}_{n^{\prime}}-\boldsymbol{u}_{n}}\right\} .
\end{aligned}
$$

Then the gradient of $\boldsymbol{s}^{*}$ with respect to the $a_{k n}$ coefficient is 


$$
\begin{aligned}
\frac{\partial \boldsymbol{s}^{*}}{\partial a_{k n}}= & -j \sum_{\substack{n^{\prime}=1 \\
n^{\prime} \neq n}}^{N_{T}}\left\{\left[\mathcal{Z}_{k} \boldsymbol{\Lambda} \exp \left(-j \sum_{k^{\prime}=1}^{3} \alpha_{n n^{\prime}}^{k^{\prime}} \mathcal{Z}_{k^{\prime}}\right)\right] \star \delta_{\boldsymbol{u}_{n}-\boldsymbol{u}_{n^{\prime}}}\right. \\
& \left.+\left[\boldsymbol{\epsilon}_{k} \mathcal{Z}_{k} \boldsymbol{\Lambda} \exp \left(-j \sum_{k^{\prime \prime}=1}^{3} \alpha_{n^{\prime} n}^{k^{\prime \prime}} \mathcal{Z}_{k^{\prime \prime}}\right)\right] \star \delta_{\boldsymbol{u}_{n^{\prime}}-\boldsymbol{u}_{n}}\right\},
\end{aligned}
$$

with $\epsilon_{k}=1$ if $\boldsymbol{Z}_{k}$ is of odd radial order and $\epsilon_{k}=-1$ otherwise because of Eq. (7).

Let us call $\mathcal{D}_{n n^{\prime}}$ the support of the frequency peak corresponding to the baseline vector $n n^{\prime}$. As the pupil configuration is diluted and nonredundant, the product of the OTF by its conjugated derivative is nonzero only on $\mathcal{D}_{n n^{\prime}}$ and $\mathcal{D}_{n^{\prime} n}$ domains. Moreover, this product is imaginary as the exponential terms simplify:

$$
\boldsymbol{s}_{\frac{\partial \boldsymbol{s}^{*}}{\partial a_{k n}}}=-j \sum_{\substack{n^{\prime}=1 \\ n^{\prime} \neq n}}^{N_{T}}\left(\mathcal{Z}_{k} \boldsymbol{\Lambda}^{2} \star \delta_{\boldsymbol{u}_{n}-\boldsymbol{u}_{n^{\prime}}}+\epsilon_{k} \mathcal{Z}_{k} \boldsymbol{\Lambda}^{2} \star \delta_{\boldsymbol{u}_{n^{\prime}}-\boldsymbol{u}_{n}}\right) .
$$

As the real part of this expression is null, Eq. (13) becomes

$$
\frac{\partial L^{\prime}}{\partial a_{k n}}=-\frac{1}{\sigma^{2}} \sum_{\boldsymbol{u} \in \mathcal{D} \backslash \mathcal{D}_{\mathbf{0}}} \mathfrak{R}\left\{\tilde{\boldsymbol{i}}(\boldsymbol{u}) \frac{\partial \boldsymbol{s}^{*}}{\partial a_{k n}}(\boldsymbol{u})\right\} .
$$

The aberrations solution verifies the equation

$$
\forall(k, n), \quad \frac{\partial L^{\prime}}{\partial a_{k n}}\left(\widehat{\alpha_{k n}}\right)=0 .
$$

Then we explicitly express the gradient as in Eq. (15):

$$
\begin{gathered}
\sum_{\boldsymbol{u} \in \mathcal{D} \backslash \mathcal{D}_{\mathbf{0}}} \mathfrak{I}\left\{\tilde{\boldsymbol{i}} \sum_{n^{\prime}=1}^{N_{T}}\left[\mathcal{Z}_{k} \boldsymbol{\Lambda} \exp \left(-j \sum_{k^{\prime}=1}^{3} \widehat{\alpha_{n n^{\prime}}^{k^{\prime}}} \mathcal{Z}_{k^{\prime}}\right)\right] \star \delta_{\boldsymbol{u}_{n}-\boldsymbol{u}_{n^{\prime}}}\right. \\
\left.+\left[\boldsymbol{\epsilon}_{k} \mathcal{Z}_{k} \boldsymbol{\Lambda} \exp \left(-j \sum_{k^{\prime \prime}=1}^{3} \widehat{\alpha_{n n^{\prime}}^{k^{\prime \prime}}} \mathcal{Z}_{k^{\prime \prime}}\right)\right] \star \delta_{\boldsymbol{u}_{n^{\prime}}-\boldsymbol{u}_{n}}\right\}(\boldsymbol{u})=0 .
\end{gathered}
$$

Using the Hermitian symmetry of $\tilde{\boldsymbol{i}}$, the centrosymmetry of $\mathcal{Z}_{k}$, and the sign of $\epsilon_{k}$, it can be shown that the second term of the sum is equal to the first one. As a consequence, only half the peaks need to be considered when looking for the estimates, and we have

$$
\begin{aligned}
& \forall n, \quad \sum_{\boldsymbol{u} \in \mathcal{D} \backslash \mathcal{D}_{\mathbf{0}}} \mathfrak{I}\left\{\tilde { \boldsymbol { i } } \sum _ { \substack { n ^ { \prime } = 1 \\
n ^ { \prime } \neq n } } ^ { N _ { T } } \left[\mathcal { Z } _ { k } \boldsymbol { \Lambda } \operatorname { e x p } \left(-j \sum_{k^{\prime}=1}^{3}\right.\right.\right. \\
& \left.\left.\left.\times \widehat{\alpha_{n n^{\prime}}^{k^{\prime}}}, \mathcal{Z}_{k^{\prime}}\right)\right] \star \delta_{\boldsymbol{u}_{n^{-}} \boldsymbol{u}_{n^{\prime}}}\right\}(\boldsymbol{u})=0 \text {. }
\end{aligned}
$$

The following two sections distinguish the specific case where pistons are the only aberrations on the subapertures from the more general case of pistons mixed with tip/tilts. In both cases we will show that the subaperture aberration $a_{k n}$ can be retrieved from the sum $\beta_{n}^{k}$ defined by

$$
\beta_{n}^{k}=\sum_{\substack{n^{\prime}=1 \\ n^{\prime} \neq n}}^{N_{T}} \alpha_{n n^{\prime}}^{k}
$$

and from the average aberration $a_{k}$ over the subapertures:

$$
a_{k}=\frac{1}{N_{T}} \sum_{n^{\prime}=1}^{N_{T}} a_{k n^{\prime}}
$$

\section{FUSCHIA for the Piston-Only Case}

Let us consider the case when aberrations are only pistons. Using Eq. (7), Eq. (21), and Eq. (22) with $\epsilon_{k}=-1, \beta_{n}^{1}$ is

$$
\beta_{n}^{1}=N_{T}\left(a_{1 n}-a_{1}\right)
$$

Then the aberration $a_{1 n}$ is

$$
a_{1 n}=a_{1}+\beta_{n}^{1} / N_{T}
$$

While $a_{1}$ is unknown and cannot be determined, this is not a problem for the purpose of MAOT cophasing, as the knowledge of a global piston is not relevant. In practice, $a_{1}$ is arbitrarily fixed at a convenient value, generally zero.

To retrieve the aberration $a_{1 n}, \beta_{n}^{1}$ has to be computed. This will be done by computing the coefficients $\alpha_{n n^{\prime}}^{1}$ using Eq. (20). Note that as Eq. (6) and Eq. (15) are exact, Eq. (20) is. It is also further simplified as the function $\mathcal{Z}_{1}$ is real, constant, and equal to unity. When aberrations are only pistons, Eq. (20) then simply becomes

$$
\forall n, \quad \sum_{\substack{n^{\prime}=1 \\ n^{\prime} \neq n}}^{N_{T}} \mathfrak{I}\left\{\exp \left(-\overline{\alpha_{n n^{\prime}}^{\top}}\right) \sum_{\boldsymbol{u} \in \mathcal{D}_{n n^{\prime}}} \tilde{\boldsymbol{i}}(\boldsymbol{u}) \Lambda\left(\boldsymbol{u}-\boldsymbol{u}_{n}+\boldsymbol{u}_{n^{\prime}}\right)\right\}=0 .
$$

As all $\mathcal{D}_{n n^{\prime}}$ are nonoverlapping frequency domains, this means the sum is null at all frequencies if and only if all its terms are

$$
\begin{aligned}
\forall n, \forall n^{\prime} \neq & n \quad \operatorname{Arg}\left[\exp \left(-j \widehat{\alpha_{n n^{\prime}}^{\top}}\right) \sum_{\boldsymbol{u} \in \mathcal{D}_{n n^{\prime}}} \tilde{\boldsymbol{i}}(\boldsymbol{u})\right. \\
& \left.\times \boldsymbol{\Lambda}\left(\boldsymbol{u}-\boldsymbol{u}_{n}+\boldsymbol{u}_{n^{\prime}}\right)\right]=0 \quad[\bmod \pi],
\end{aligned}
$$

which becomes

$$
\begin{aligned}
\forall n, \forall n^{\prime} \neq & n-\widetilde{\alpha_{n n^{\prime}}^{\top}}+\operatorname{Arg}\left[\sum_{\boldsymbol{u} \in \mathcal{D}_{n n^{\prime}}} \tilde{\boldsymbol{i}}(\boldsymbol{u})\right. \\
& \left.\times \Lambda\left(\boldsymbol{u}-\boldsymbol{u}_{n}+\boldsymbol{u}_{n^{\prime}}\right)\right]=0 \quad[\bmod \pi] .
\end{aligned}
$$

Using the result of Eq. (24), $\hat{a}_{1 n}$ is finally given by 


$$
\begin{aligned}
\hat{a}_{1 n}= & \frac{1}{N_{T}} \sum_{\substack{n^{\prime}=1 \\
n^{\prime} \neq n}}^{N_{T}} \operatorname{Arg}\left[\sum_{\boldsymbol{u} \in \mathcal{D}_{n n^{\prime}}} \tilde{\boldsymbol{i}}(\boldsymbol{u})\right. \\
& \left.\times \boldsymbol{\Lambda}\left(\boldsymbol{u}-\boldsymbol{u}_{n}+\boldsymbol{u}_{n^{\prime}}\right)\right] \quad[\bmod 2 \pi] .
\end{aligned}
$$

In Eq. (28), only the modulo $2 \pi$ solutions are kept since the other solutions of Eq. (18) correspond to maxima of the $L^{\prime}$ criterion when $\boldsymbol{s}$ and $\tilde{\boldsymbol{i}}$ are phase opposite, as demonstrated by Eq. (12). Therefore, there is only a single solution in the nonambiguous piston range $]-\pi, \pi]$ for the piston on each subaperture $n$, explicitly given as a function of the image. Let us recall that $i$ is the image of an unresolved source, so $\widetilde{\boldsymbol{i}}$ is very close to the OTF $\boldsymbol{s}$. Thus the physical meaning of Eq. (28) is that the phase is extracted from each OTF peak with a weighting factor equal to the modulus $\Lambda$ of an unaberrated peak. This means in particular a higher phase contribution near the center of the peak, where the signal-to-noise ratio is higher.

\section{FUSCHIA for Mixed Pistons and Tilts}

With aberrations other than pistons, the phase terms within the exponential of Eq. (20) vary over $\mathcal{D}_{n n^{\prime}}$. For small aberrations however, the exponential can be expanded into a Taylor series. Equation (6) shows that the modulus of $\tilde{\boldsymbol{i}}$ on each peak is equal to $\boldsymbol{\Lambda}$ on the first order. Let $\Phi$ be the phase of $\tilde{\boldsymbol{i}}$. Then Eq. (20) becomes

$$
\begin{aligned}
\forall n, \quad \sum_{\substack{n^{\prime}=1 \\
n^{\prime} \neq n}}^{N_{T}} \sum_{\boldsymbol{u} \in \mathcal{D}_{n n^{\prime}}} \mathfrak{I}\left\{\left[\boldsymbol{\Lambda}^{2} \mathcal{Z}_{k}\right]\left(\boldsymbol{u}-\boldsymbol{u}_{n}+\boldsymbol{u}_{n^{\prime}}\right)\right. \\
\left.\quad \times\left[1+j \Phi(\boldsymbol{u})-j \sum_{k^{\prime}=1}^{3} \hat{\alpha}_{n n^{\prime}}^{k^{\prime}}, \mathcal{Z}_{k^{\prime}}\left(\boldsymbol{u}-\boldsymbol{u}_{n}+\boldsymbol{u}_{n^{\prime}}\right)\right]\right\} \approx 0,
\end{aligned}
$$

which simplifies into

$$
\begin{aligned}
& \forall n, \quad \sum_{\substack{n^{\prime}=1 \\
n^{\prime} \neq n}}^{N_{T}} \sum_{\boldsymbol{u} \in \mathcal{D}_{n n^{\prime}}} \Phi(\boldsymbol{u})\left[\Lambda^{2} \mathcal{Z}_{k}\right]\left(\boldsymbol{u}-\boldsymbol{u}_{n}+\boldsymbol{u}_{n^{\prime}}\right) \\
& n^{\prime} \neq n \\
& \approx \sum_{n^{\prime}=1}^{N_{T}} \sum_{k^{\prime}=1}^{3} \hat{\alpha}_{n n^{\prime}}^{k^{\prime}}\left[\sum_{\boldsymbol{u} \in \mathcal{D}_{n n^{\prime}}}\left[\boldsymbol{\Lambda}^{2} \mathcal{Z}_{k} \mathcal{Z}_{k^{\prime}}\right]\left(\boldsymbol{u}-\boldsymbol{u}_{n}+\boldsymbol{u}_{n^{\prime}}\right)\right] \text {. }
\end{aligned}
$$

The integral of $\Lambda^{2} \mathcal{Z}_{k} \mathcal{Z}_{k^{\prime}}$ on each frequency domain $\mathcal{D}_{n n^{\prime}}$ is independent of $n$ or $n^{\prime}$. Even with the $\Lambda^{2}$ weighting, $\mathcal{Z}_{1}, \mathcal{Z}_{2}, \mathcal{Z}_{3}$ is still an orthogonal basis, so that $\Lambda^{2} \mathcal{Z}_{k} \mathcal{Z}_{k^{\prime}}$ is nonzero if and only if $k^{\prime}=k$. Thus Eq. (30) allows us to estimate $\beta_{n}^{k}$, defined in Eq. (21), as

$$
\begin{aligned}
& \forall n, \quad \widehat{\beta_{n}^{k}}=\sum_{n^{\prime}=1}^{N_{T}} \hat{\alpha}_{n n^{\prime}}^{k} \\
& n^{\prime} \neq n \\
& \sum_{n^{\prime}=1}^{N_{T}} \sum_{\boldsymbol{u} \in \mathcal{D}_{n n^{\prime}}} \Phi(\boldsymbol{u})\left[\boldsymbol{\Lambda}^{2} \mathcal{Z}_{k}\right]\left(\boldsymbol{u}-\boldsymbol{u}_{n}+\boldsymbol{u}_{n^{\prime}}\right) \\
& \approx \frac{n^{\prime} \neq n}{N_{T}} \\
& \sum_{n^{\prime}=1}^{N_{T}} \sum_{\boldsymbol{u} \in \mathcal{D}_{n n^{\prime}}}\left[\boldsymbol{\Lambda}^{2} \mathcal{Z}_{k}^{2}\right]\left(\boldsymbol{u}-\boldsymbol{u}_{n}+\boldsymbol{u}_{n^{\prime}}\right) \\
& n^{\prime} \neq n
\end{aligned}
$$

From $\beta_{n}^{k}$ the pistons can be retrieved as described Subsection 3.B.2. Since the tips or tilts are Zernike modes of odd radial order, Eq. (7) implies that

$$
\beta_{n}^{k}=\left(N_{T}-2\right) a_{k n}+N_{T} a_{k} .
$$

To compute $a_{k}$ we perform a closure by summing all $\beta_{n^{\prime}}^{k}$ on all apertures $n^{\prime}$ :

$$
\sum_{n^{\prime}=1}^{N_{T}} \beta_{n^{\prime}}^{k}=2 N_{T}\left(N_{T}-1\right) a_{k} .
$$

Finally, the aberration $k$ on the subaperture $n$ is given by

$$
\hat{a}_{k n}=\frac{\widehat{\beta_{n}^{k}}}{N_{T}-2}-\frac{1}{2\left(N_{T}-1\right)\left(N_{T}-2\right)} \sum_{n^{\prime}=1}^{N_{T}} \widehat{\beta_{n^{\prime}}^{k}} .
$$

Equations (31) and (34) allow us to retrieve all tip/tilt aberrations on each subaperture without ambiguity. One of FUSCHIA's major strengths resides in the fact that Eqs. (28), (34), and (31) require the computation of only one FFT to retrieve both the pistons and the tips/tilts. Consequently FUSCHIA brings a major speed improvement over the iterative solution, being on average 20 times faster.

Equation (34) is defined only if $N_{T} \geq 3$. This may seem a rather surprising result since $N_{T}=2$ allows one to measure piston, as demonstrated by Eq. (24) and well established with the Young setup. This results from the fact that for $N_{T}=2$ the pupil is centrosymmetric, and so the even part of the phase cannot be retrieved. This raises no problem for piston, as the average piston (even mode) is already known to be not seen and thus is not solved for. But the differential piston (odd mode) can be correctly estimated. A similar situation occurs for tip/tilt, which can be better illustrated from the focal-plane data: If a similar tip/tilt is applied on both apertures, then both PSFs shift by the same amount, leading to a global linear phase in the OTF argument. But if opposite tips/tilts are applied, then two identical separated PSFs are observed. It is possible to derive the absolute value of the tip/tilt from the distance between the two PSFs. More precisely, this measurement involves only the OTF modulus, since the image is also centrosymmetric and thus the phase of the OTF is zero. But it is not possible to guess to which aperture belongs each PSF from the sole focal-plane image and thus to estimate the tip/tilt sign. The first example shows that the average tip/tilt (coefficient $\alpha_{12}^{k+}, k \in\{1,2\}$ in Appendix A) can be estimated, since it is linked to an odd mode, and 
the second example confirms that the sign of the differential tilt (coefficient $\alpha_{12}^{k-}, k \in\{1,2\}$ ) cannot be estimated, because the differential tip/tilt is an even mode. The full measurement was possible in the first example because there was no differential tip/tilt. Since the general case is a combination of average and differential tips/tilts, the lack of the differential estimation prevents us from estimating the individual tips/tilts.

\section{Case of Compact Pupil Configurations}

So far we have assumed that the peaks in the OTF do not overlap. This restriction means in practice that some spatial frequencies will be missed by the MAOT, which is bad for instantaneous imaging of extended objects. However, the instrument may take advantage of aperture synthesis by rotation to complete its frequency coverage. Many interferometers are also not designed primarily for direct imaging purposes and rely on image reconstruction software.

In practice, however, one may have to work with an instrument whose pupil configuration has its OTF peaks overlapping. This may arise in two cases. The first case is when the pupil is redundant. More than one pair of apertures then form an identical baseline, thus contributing to the exact same spatial frequency peak. The phases cannot then be easily disentangled (FUSCHIA would not be used). The second case is when peaks overlap but not totally. This happens in particular for compact pupil configurations meant for imaging, whose OTFs do not reach zero inside defined frequency domains of interest. As FUSCHIA is dedicated to cophasing or fringe-tracking instruments and is not directly used for science measurements, the pupil can sometimes be optically remapped into a diluted pupil. If not, it may still be possible to use FUSCHIA, though not optimally. If the peak overlap takes place in the outer periphery of the peaks, then the central unambiguous part of the peaks can be extracted and used with success in place of the whole peaks. Our experimental data in Section 5 were in fact processed with this procedure.

\section{NUMERICAL VALIDATIONS}

In this section are presented the results of simulations comparing both LS estimators (FUSCHIA and the iterative retrieval method). The typical performance in closed and open loop is analyzed, as well as the algorithm linearity when confronted by piston and tip/tilt ramps.

\section{A. Conditions of Simulations}

A complete simulation environment has been developed to validate and to compare both estimators. It is able to compute the focal-plane image of any observed object viewed through an interferometric instrument of a given input pupil.

All simulations are performed with a wavelength $\lambda$ of $650 \mathrm{~nm}$. The object is a point source, while the chosen pupil, shown on Fig. 2, is an equilateral triangle. The dilution factor, defined as the shortest baseline divided by the subaperture diameter, is chosen equal to 2 (the minimum dilution for such a diluted pupil). This choice reflects a compromise between the need to have a diluted pupil for the analytical algorithm to work optimally and maintaining a decent spatial frequency coverage.

Images with $64 \times 64$ pixels are simulated at increasing fluxes. As the object is a point source, most of the flux is concentrated on a small zone of the image. Only this zone of interest (typically $14 \times 14$ pixels) is used for analysis. The total flux per frame ranges from $10^{2}$ to $10^{7}$ photoelectrons. Photon noise is added, as well as a typical read-out noise of standard deviation $\sigma_{d}=10.0$ electrons per pixel. One hundred images are simulated for every flux, each one corresponding to a different noise outcome. Both estimators are fed these images as well as the input pupil. Note that as the iterative estimator is meant to be used for cophasing purposes, the starting aberrations for its initialization are chosen null in all cases. The estimator computes the subaperture aberration estimates. Finally, the statistical errors (standard deviations and mean errors) are computed for each aberration on each subaperture.

\section{B. Results and Analysis}

\section{Linearity}

The linearity results, obtained from piston and tilt ramps of $[-\lambda, \lambda]$ with a flux of $10^{5}$ photoelectrons per frame, are presented on Fig. 3.

For piston, both estimators behave linearly on the domain $[-\lambda / 2, \lambda / 2]$, with a wrapping of $\lambda$ when the modulus of the amplitude increases beyond $\lambda / 2$. This is a classical result for monochromatic piston estimation. On each linear part the slope for both curves is unity. As no approximation is realized in the piston-only case, FUSCHIA and the iterative estimator produce identical results (to machine precision), as expected. In contrast, in the tilt linearity test, while the iterative estimator is linear on the whole domain $[-\lambda / 2, \lambda / 2]$, FUSCHIA remains linear only on a limited domain that roughly corresponds to [ $-0.3 \lambda, 0.3 \lambda]$. The main reason for the shape of the plot outside this domain is not the failure of the small-phase approximation (though this plays a minor role) but phase wrapping. The phase of the OTF is indeed explicitly extracted by a very simple routine (arctangent). As the amplitude of the tilt mode across an aperture is $4 a_{k n}$, when $a_{k n}$ is greater than $\lambda / 4$, the amplitude exceeds $\lambda$. The phase derived by arctangent is then partially wrapped. The implementation of an unwrapping procedure is planned for future simulations. Note that the iterative estimator does not suffer from this problem, as the phase is not explicitly extracted by the algorithm. In practice the domain limitation for the tilt estimation should not constitute a major problem when cophasing an instrument, as the aberrations to correct should already be smaller than $0.3 \lambda$.

FUSCHIA for tilts requires the validity of the smallphase approximation; thus the slope of FUSCHIA's curve within the linear domain is not exactly unity. Figure 3(c) shows the remaining bias to be smaller than $\lambda / 80$. For a cophasing system in closed loop this implies only that more FUSCHIA iterations will be needed to correct the aberrations. This might, however, constitute an issue in open loop. 


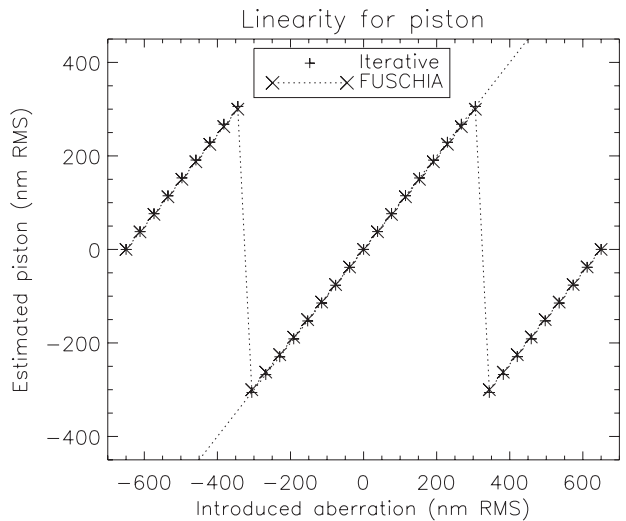

(a)

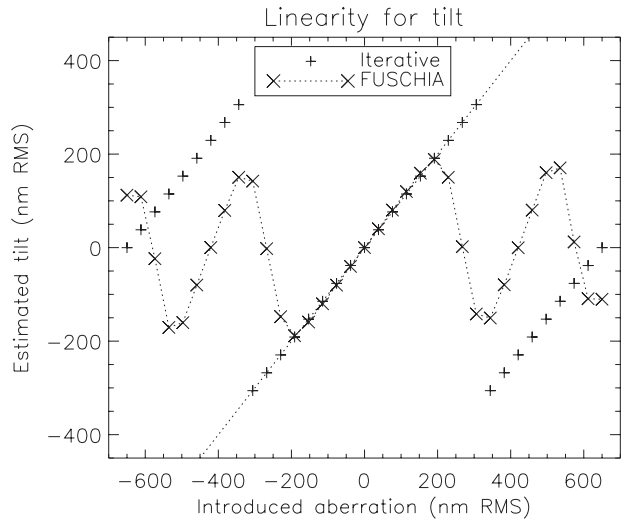

(b)

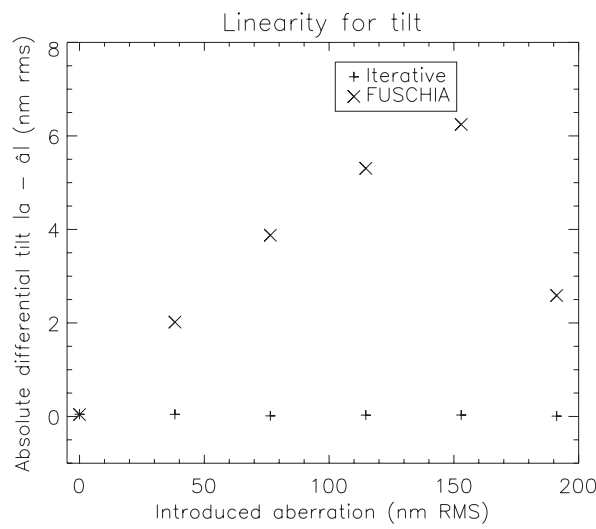

(c)

Fig. 3. Responses of FUSCHIA and of the iterative estimator to a piston ramp (a) and a tilt ramp (b). The tilt estimation is slightly biased for FUSCHIA in the linear domain due to the approximated nature of the algorithm (c).

\section{Performance and Comparison of the Two Estimators} Figures 4 and 5 allow us to examine the accuracy (error) and repeatability (standard deviation) of each estimator in the absence of aberrations. The performance in such a case is representative of what could be expected in a closed loop.

The mean error and the standard deviation curves are extremely similar, as the bias is very low. Within our flux range the bias is insignificant (zero) in the piston estimation test, and it remains under $0.1 \mathrm{~nm}$ for fluxes higher than $10^{4}$ photoelectrons per frame in the tilt estimation test. The repeatability performance is very good, as the
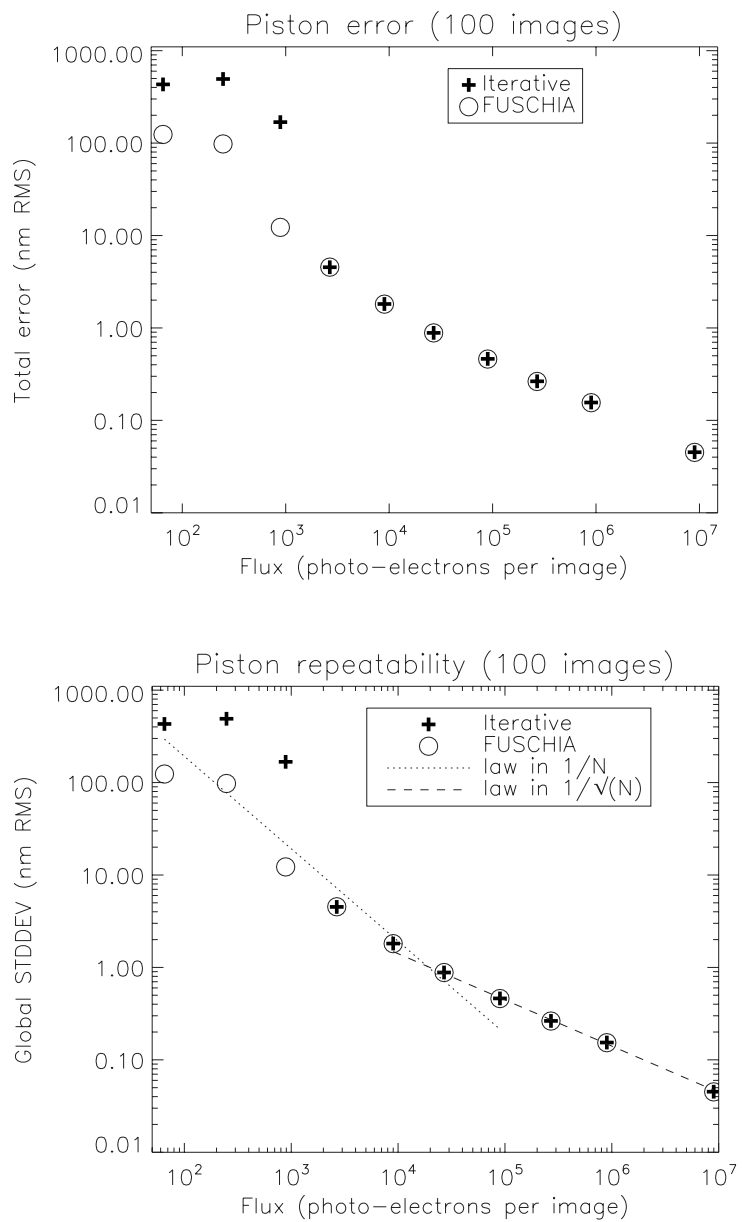

Fig. 4. Error and standard deviation on piston estimates for FUSCHIA and the iterative estimator (no aperture aberrations).

standard deviations are below $1.0 \mathrm{~nm} \mathrm{rms}$ for fluxes higher than $2 \times 10^{4}$ photoelectrons per frame in the piston case and $10^{5}$ photoelectrons per frame in the tilt case.

The shape of the standard deviation curves is typical of this type of phase estimation. Within the flux range of $\left[10^{2}, 10^{7}\right]$ photoelectrons per frame chosen for our figures, the standard deviation $\sigma$ (in nanometers) theoretically follows the equation [33]:

$$
\sigma=\frac{\lambda}{2 \pi} \frac{\gamma}{\operatorname{SNR} \sqrt{n_{\text {pix }}}}
$$

where the $\gamma$ coefficient is a constant coefficient expressed in radians measuring the global performance of the algorithm, $n_{\text {pix }}$ the number of pixels per frame (here $14 \times 14$ ), and SNR is the signal-to-noise-ratio per pixel defined by

$$
\mathrm{SNR}=\frac{N / n_{\text {pix }}}{\sqrt{N / n_{\text {pix }}+\sigma_{d}^{2}}},
$$

with $N$ the total flux per frame in photoelectrons. The relative importance of the parameters $N / n_{\text {pix }}$ and $\sigma_{d}$ determines the regime of the estimator. When the flux is lower than $1.3 \times 10^{4}$ photoelectrons per frame, the noise is dominated by the detector noise and $\sigma$ is proportional to $1 / N$. For fluxes higher than $1.3 \times 10^{4}$ photoelectrons per frame, the Poissonnian photon noise becomes dominant 

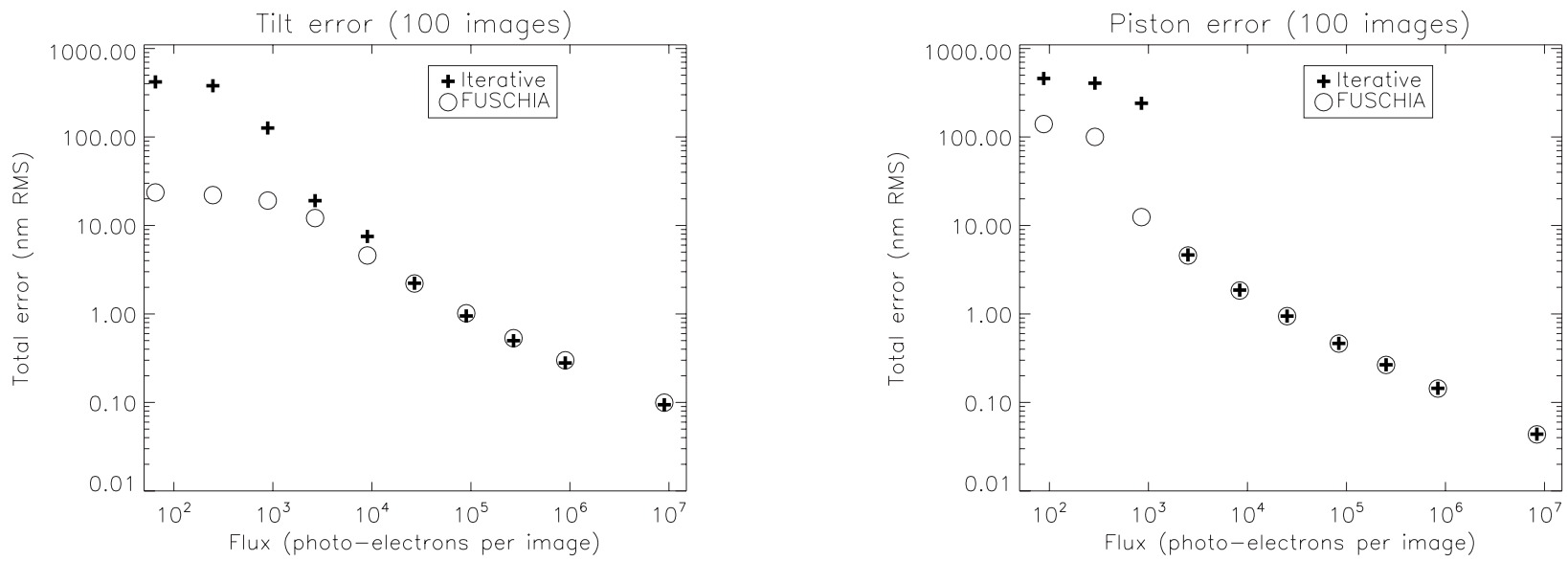

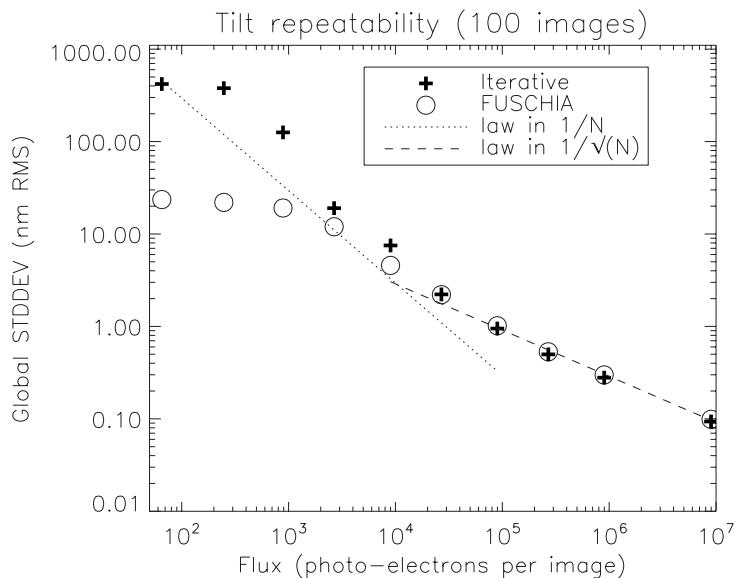

Fig. 5. Error and standard deviation on tilt estimates for FUSCHIA and the iterative estimator (no aperture aberrations).

and $\sigma$ is proportional to $1 / \sqrt{N}$. This change of slope from 1 to $1 / 2$ is especially visible on Fig. 4 (b). The $\gamma$ coefficients derived in the photon regime range are identical for the two estimators, about $1.3 \mathrm{rad}$ on the piston curves and $2.6 \mathrm{rad}$ on the tilt ones. These results prove that tilt is generally more difficult to estimate than piston. They also confirm that the analytical estimator performs similarly to the iterative one for cophasing purposes. The behavior of both estimators in open loop is depicted by Fig. 6 (resp. Fig. 7), which corresponds to simulations done with a piston (resp. a tilt) of $\lambda / 6$. On one hand, the piston estimations are nearly unchanged, and the $\gamma$ coefficient remains at 1.3 for both estimators.

On the other hand, the tilt results show a strong discrepancy between the estimators. As underlined previously in the linearity test, the bias of the analytical estimator is not zero in open loop. Figure 7(c) shows that as the flux increases, the bias of the analytical estimator reaches a plateau at $1.0 \mathrm{~nm}$. This results in a nearly constant mean error of about $2 \mathrm{~nm}$ for fluxes higher than 2 $\times 10^{4}$ photoelectrons per frame. The error of the iterative estimator, however, behaves as in the previous case, going as low as $0.01 \mathrm{~nm}$ at $10^{7}$ photoelectrons per frame. The repeatabilities are indeed still very good for both estimators, as the $\gamma$ coefficient for the tilt curve is 2.2 for the iterative estimator, though it goes up to about 3.3 for FUSCHIA.

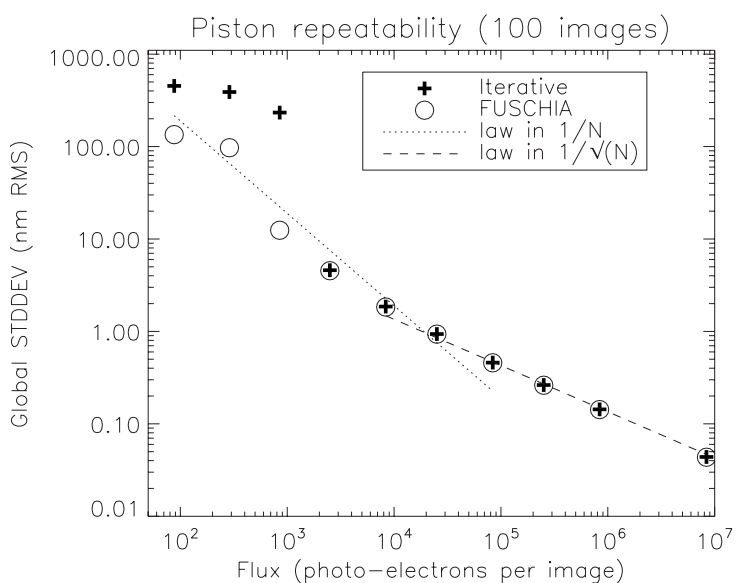

Fig. 6. Error and standard deviation on piston estimates for FUSCHIA and the iterative estimator (aperture aberrations of $\lambda / 6)$.

Thus, overall, the iterative estimator is more adapted to open-loop systems, while FUSCHIA is particularly suited to closed-loop ones.

\section{EXPERIMENTAL VALIDATION}

\section{A. BRISE Bench}

In order to evaluate the performance of focal-plane cophasing sensors, ONERA has built a multipurpose bench called BRISE [34,35] (Banc Reconfigurable d'Imagerie sur Scènes Etendues). It includes mainly a segmented mirror that can introduce calibrated piston/ tip/tilt aberrations, a focal-plane sensor, and both an extended scene and a reference point source.

The experimental validation of the iterative and the FUSCHIA estimators was done with the BRISE internal wavefront sensor and with DARWIN's fringe sensor breadboard, DWARF, installed on the bench [DARWIN is a future European Space Agency (ESA) mission dedicated to the search for life signs on Earthlike planets]. The concept, selected by ONERA, is based on a combination telescope that focuses the three beams of the instrument on a focal-plane camera. Differential piston and tip/tilt are estimated with phase retrieval algorithms. The breadboard we used on the bench was developed for ESA by KayserThrede/ONERA/Alcatel Alenia Space [36,37]. In Subsec- 


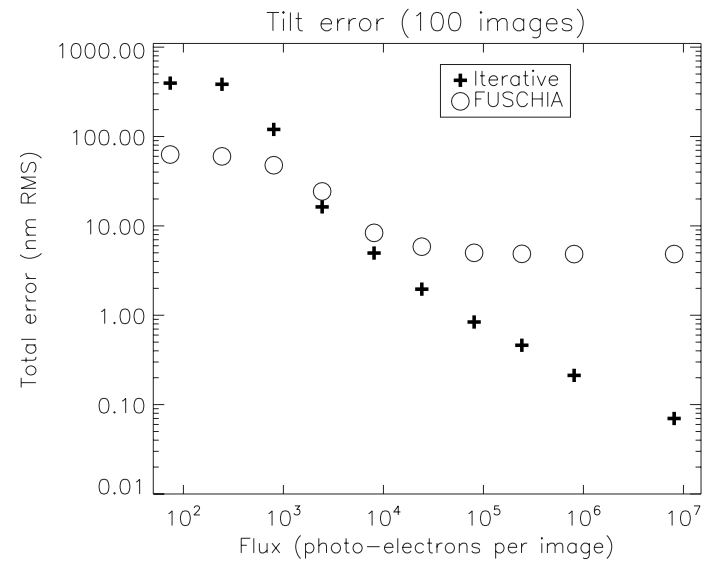

(a)

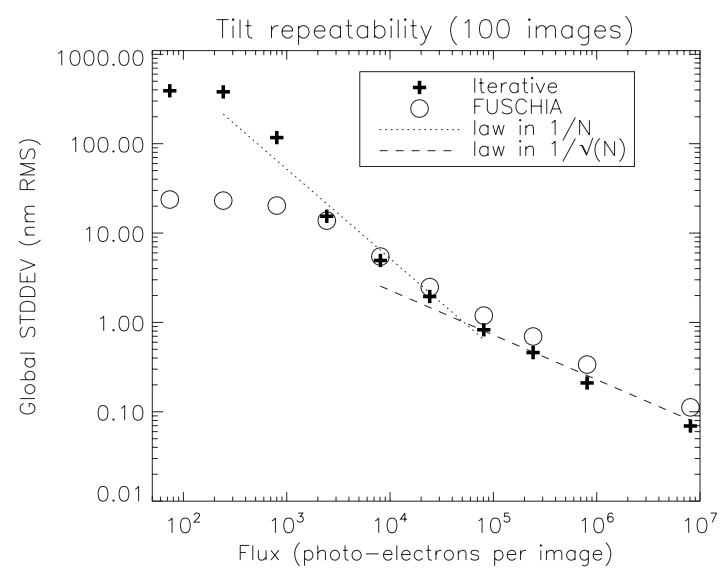

(b)

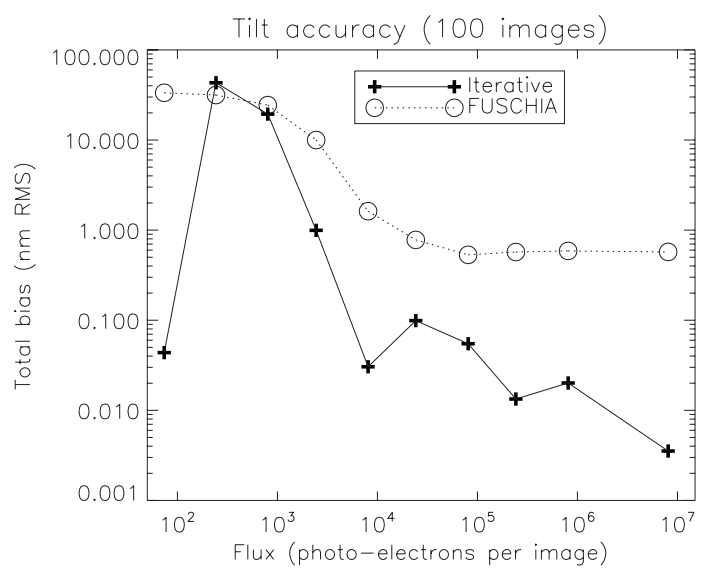

(c)

Fig. 7. Error, standard deviation, and bias on tilt estimates for FUSCHIA and the iterative estimator (aperture aberrations of $\lambda / 6)$.

tions 5.B and 5.D we present critical steps for the test-bed calibration before we detail in Subsections $5 . \mathrm{E}$ and $5 . \mathrm{F}$ the experimental results obtained with DWARF on BRISE.

\section{B. Accordance between the Numerical and the Physical Models}

The direct model allows image simulation from the known experimental parameters (object, pupil configuration, wavelength, etc). It is used to model the experiment, par- ticularly to evaluate the influence of the various noises and experimental parameters on the cophasing sensor performance. But it is also used in the phase-estimation algorithms for the resolution of the inverse problem (phase reconstruction from the image). Therefore, the direct model must be as close as possible to the physical model; the previously listed parameters must be estimated accurately. Since the most critical parameter is the numerical pupil, i.e., the $2 \mathrm{D}$ support in pixels over which the Zernike phase screens are applied, it must match the experimental one. Its estimation at a given wavelength is made by a LS fit between the experimental OTF derived from the image $\boldsymbol{i}$ of the nearly unaberrated source and a theoretical OTF depending on parameters due to alignment and sampling defaults.

The numerical pupil can then be used for both image simulation and aberration estimation. Thus, to verify the accordance between the direct and experimental models, we apply a sequence of Zernike mode of interest on a given subaperture (as illustrated Fig. 8), and we compare the images obtained by simulation and by experiment. First, in the first row, images are taken without aberration. Then, in the second row a piston of $\lambda / 2$ is introduced on a subaperture, which makes the fringes shift in the direction of this aperture. Last, in the third row, an angular tilt of $2.44 \lambda / D$ is applied on one aperture, which produces a PSF shift in the focal plane such that its first dark ring is tangent to one of the other two superimposed PSFs. The Young fringes we can see are perpendicular to their baseline.

\section{BRISE Characterization}

Another critical issue is the bench stability. Therefore, special care has been taken to control errors that could limit the performance. In order to characterize the bench

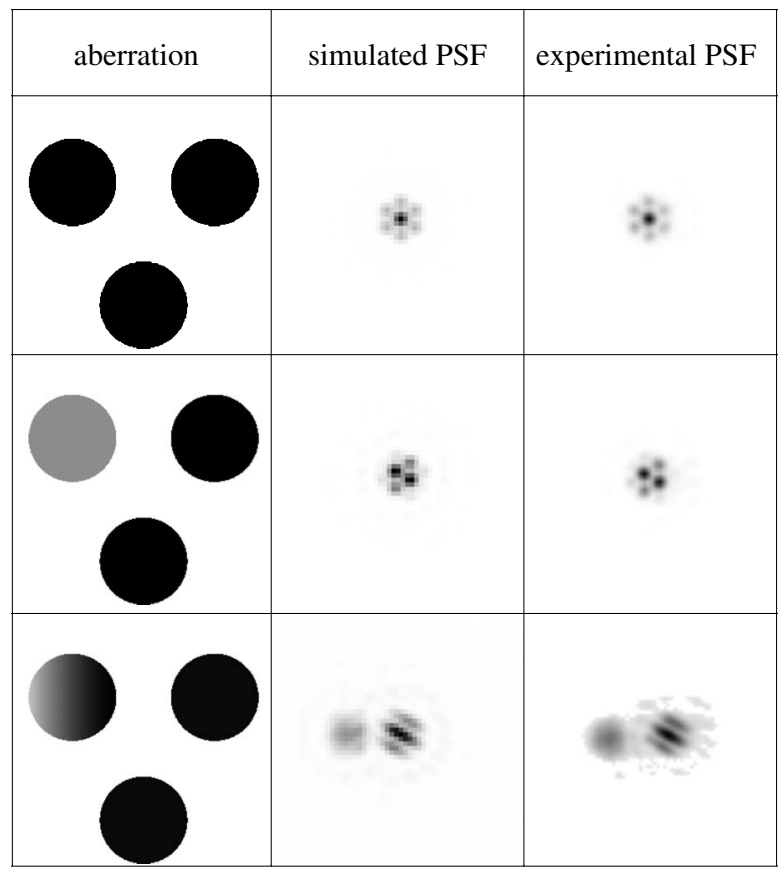

Fig. 8. Agreement between the experimental (right column) and the direct model (center column) in response to a given perturbation (left column). 
stability, we studied the temporal evolution of the estimated pistons, using both the deformable mirror and a planar mirror. The corresponding images are simultaneously recorded on the camera, as illustrated on Fig. 9(a). Measurements are made at high flux with an exposure time representative of the BRISE acquisition; estimated aberrations are computed with the FUSCHIA algorithm. Figure 9(b) plots the piston temporal evolution on the planar mirror; it shows a global tilt oscillation at $2 \mathrm{~Hz}$ with subnanometric amplitude. We can see in Fig. 9(c) that using the deformable mirror, this oscillation is perturbed due to the positioning noise introduced by the mobile mirror platforms, which is less than $3 \mathrm{~nm}$. However, in each case, there is no temporal drift, even with more significant exposure time; repeatability tests can be performed. They will be made with the planar mirror, which corresponds to the operating condition in closed loop (phase perturbation near zero) and which does not have positioning noise.

\section{Conditions of the Experiment}

For this experimental validation of both DWARF and our estimators, we have selected a noncentrosymmetric configuration, composed of three subapertures in an equilateral configuration with a dilution of 1.62 . The configuration is therefore compact. FUSCHIA was developed assuming that the OTF peaks are separated, but as underlined previously at the end of Section 2, the overlaps in the OTF will be rejected for the moment: Each peak will

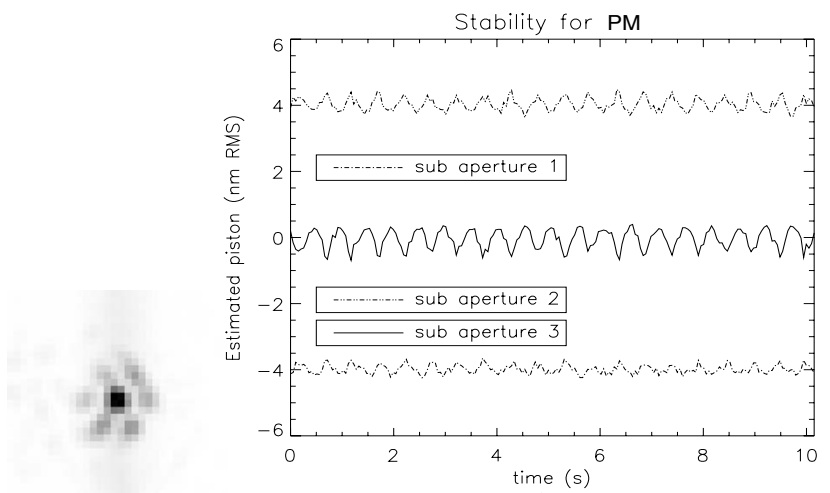

(b)

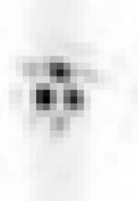

(a)

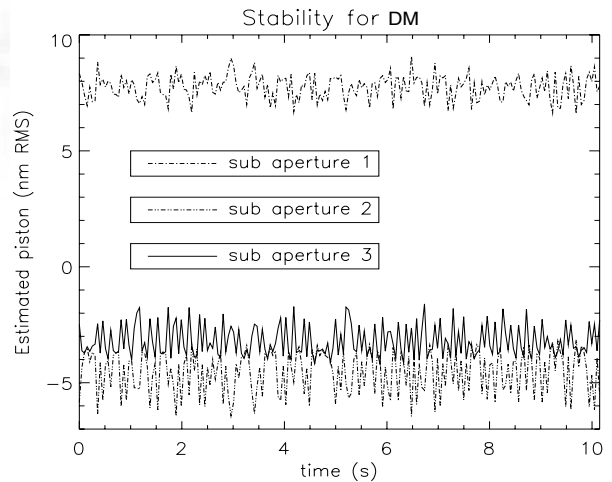

(c)

Fig. 9. Bench stability: (a) PSFs obtained with the planar (top) and deformable mirror (bottom), with the corresponding temporal evolution (b) and (c). be reduced to the largest disk inscribed in the integration domain, so that the $\mathcal{Z}_{k}$ modes calculated on this field remain mutually orthogonal.

As FUSCHIA is a closed-loop estimator, the following procedures are used to reach an initial nearly cophased state where residual pistons and tips/tilts are lower than $2 \pi \mathrm{rad}$. While the tips/tilts are uncorrected, $N_{T}$ distinct PSFs are visible on the detector, each PSF corresponding to a subaperture. Precisely superimposing all PSFs directly allows to reduce all differential tips/tilts below $2 \pi \mathrm{rad}$. Then by slowly increasing or decreasing the optical path difference associated with each subaperture, we can scan for fringes. When fringes are obtained, a conventional algorithm is used to find the central fringe by maximizing the peak modulus in the OTF. Once this fringe is secured, FUSCHIA can be used. Note that in the general case where pistons and tips/tilts fluctuate simultaneously, fringe scanning as described may become difficult, and more robust algorithms are recommended.

For all the tests presented in this section, we record small images of size $64 \times 64$ pixels at $\lambda_{c}=650 \mathrm{~nm}$, with respect to the Shannon-Nyquist sampling of the images at the focal plane. Two kinds of tests are performed:

- For the linearity test, we apply a sequence of 31 values of a single Zernike mode, piston or tilt, acquiring images at hight flux $\left(>6 \times 10^{5}\right.$ photo-electrons $)$. Because diffraction is chromatic, focal-plane images are affected by the bandwidth, whereas our model is monochromatic; the spectral band is then an important parameter to optimize. We thus perform several acquisitions, for the reference point source illuminated with the arc lamp and different spectral filters of width $10 \mathrm{~nm}$ (so-called $F_{1}$ ), $40 \mathrm{~nm}$ $\left(F_{2}\right)$, and $80 \mathrm{~nm}\left(F_{3}\right)$ centered on $\lambda_{c}$.

- In the case of the repeatability test, increasing fluxes are considered, ranging from $1.5 \times 10^{4}$ photo-electrons per image $(\mathrm{SNR}=3)$ to $1.4 \times 10^{6}$ photo-electrons $(\mathrm{SNR}=82)$. For each level, a data set of 90 images is acquired using the arc lamp and the $F_{2}$ filter. Since its flux is constant, the luminosity is changed by adjusting the exposure time, covering the whole possible dynamic range of the camera. We use the planar mirror, ensuring that we are near the zero optical path difference (OPD) and avoiding mechanical noise introduced by the piezo-electric platforms of the deformable mirror.

For each test, we first acquire a reference image without phase perturbation to estimate the numerical pupil. Then, aberrations are introduced and retrieved using both the iterative and the analytic estimators.

\section{E. Validation of Piston Estimators}

\section{Linearity}

To check the correct behavior of our phase-retrieval estimators, we first consider linearity tests, applying at high flux $(\mathrm{SNR}>70)$ a 30 -point piston ramp of $[-500 \mathrm{~nm}$, $+500 \mathrm{~nm}]$ on a given subaperture with the BRISE deformable mirror; some of the corresponding images are shown Fig. 10(a). Figure 10(b) presents, for the mobile subaperture, the piston estimated with the analytic algorithm FUSCHIA using the three filters. We note that near roughly $-\lambda_{c} / 2$ and $+\lambda_{c} / 2$, wrapping occurs due to the intrinsic modulo $2 \pi$ dynamic range of the estimator. Be- 

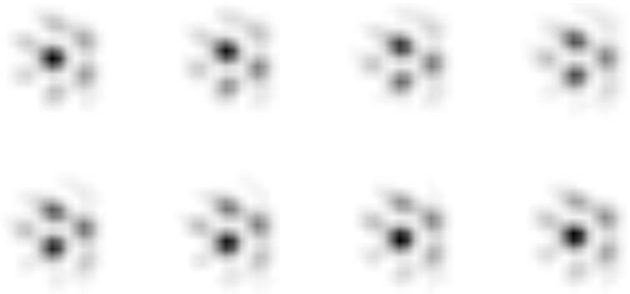

(a)

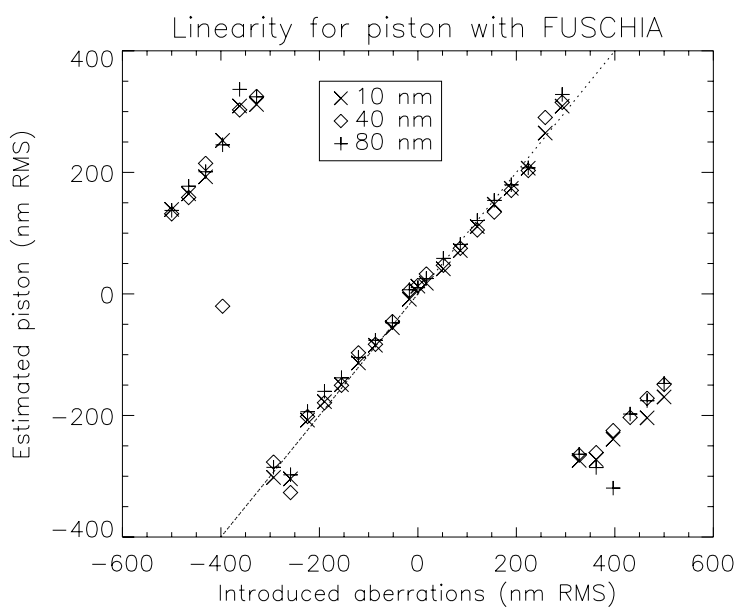

(b)

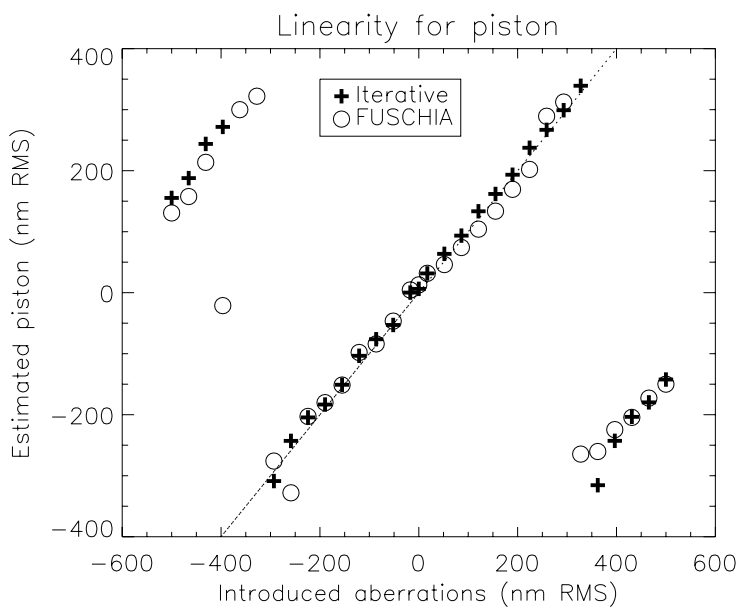

(c)

Fig. 10. Linearity of piston estimators: (a) PSFs obtained during a piston scan, from $-\lambda_{c}$ to $-\lambda_{c} / 2$; (b) piston estimated with FUSCHIA for different bandwidths; (c) comparison between FUSCHIA and the iterative algorithm for the $40 \mathrm{~nm}$ bandwidth.

tween these two wrappings, the linearity is excellent, with the slope coefficients close to 1 for each spectral band ( 0.91 for $F_{3}, 0.96$ for $F_{1}$ and $F_{2}$ ). The smallest error is obtained with $F_{1}$ : It is equal to $8 \mathrm{~nm}$, whereas it is equal to $11 \mathrm{~nm}$ and $10 \mathrm{~nm}$ for $F_{2}$ and $F 3$, respectively. But with a small band, the number of photons collected is less important, and consequently the repeatability will decrease. There is thus an optimal bandwidth, resulting from a compromise between accuracy and repeatability; we chose the $40-\mathrm{nm}$-width filter for the next tests. However, we underline that piston cophasing on large spectral band ( $80 \mathrm{~nm}$ at $650 \mathrm{~nm}$, i.e., $\Delta \lambda=\lambda_{c} / 8$ ) is possible with our monochromatic model. For much wider bandwidth, cophasing operation is possible by explicitly modeling the chromatic dependence [38].

Figure 10(c) shows the piston linearity obtained with the two focal-plane estimators, selecting the images obtained with the $40 \mathrm{~nm}$ bandwidth. In each case the piston is quite well reconstructed with slope coefficients close to 1 (0.96 for FUSCHIA, 0.99 for the iterative algorithm) as specified, since no approximation is made for piston. Realtime correction is thus possible in closed loop.

\section{Repeatability}

Figure 11 presents the piston repeatability for different levels of source brightness. For each data set, the standard deviation of estimated piston is plotted versus the flux $N$ in photo-electrons per image, after the global piston has been removed. First, we note that FUSCHIA and the iterative algorithm give identical results as expected. The graph also shows that in the photon-noise regime, the $\gamma$ coefficients of Eq. (35) are 4.5 for both estimators. We also note that piston estimation is dominated by the detector noise at the lower flux; the boundary between the two regimes lies around $1.5 \times 10^{4}$ photo-electrons.

With $\sigma_{d}=8.5$ electrons for DWARF, the number of valid pixels $n_{\text {pix }}$ is in reality equivalent to $14 \times 14$ pixels, which means that $\mathrm{SNR}=6$. Finally, the $0.75 \mathrm{~nm}$ repeatability specified for DWARF is reached with approximatively 5 $\times 10^{5}$ photo-electrons per image, which correspond to $\mathrm{SNR}=50$.

\section{F. Validation of Tilt Estimators}

\section{Linearity}

To study the tilt linearity, we apply a 30-point tilt ramp going from $-250 \mathrm{~nm}$ to $250 \mathrm{~nm}$ at high flux (SNR $>70)$; some of the PSFs obtained during the scan are illustrated on Fig. 12(a). On Fig. 12(b) we present the tilt estimated by FUSCHIA for the three spectral filters $F_{1}, F_{2}$, and $F_{3}$. First, we note that beyond $\left|\lambda_{c} / 4\right|$, the tilt is not well reconstructed because the three PSFs no longer superimpose. In the validity domain, results obtained with the different filters are similar, with a slope coefficient of 1.15 in each

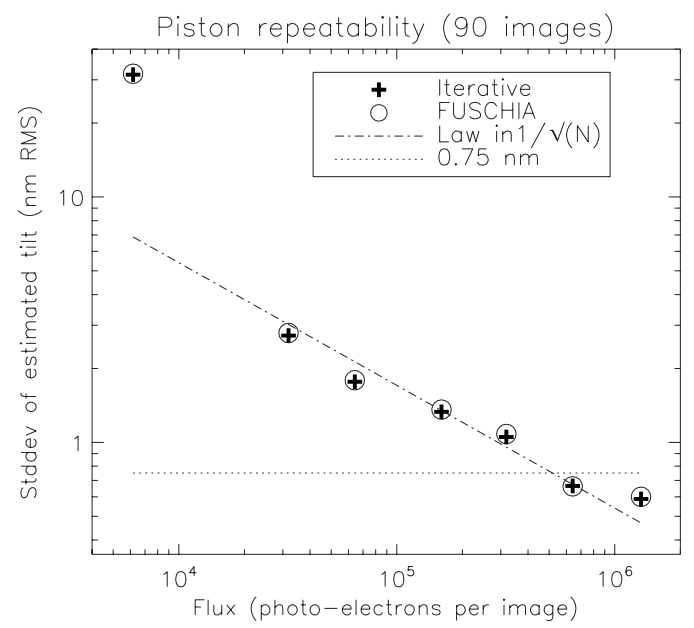

Fig. 11. Piston repeatability estimated with FUSCHIA and the iterative algorithm. 


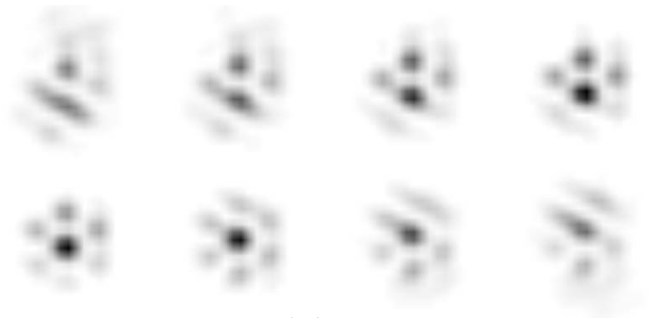

(a)

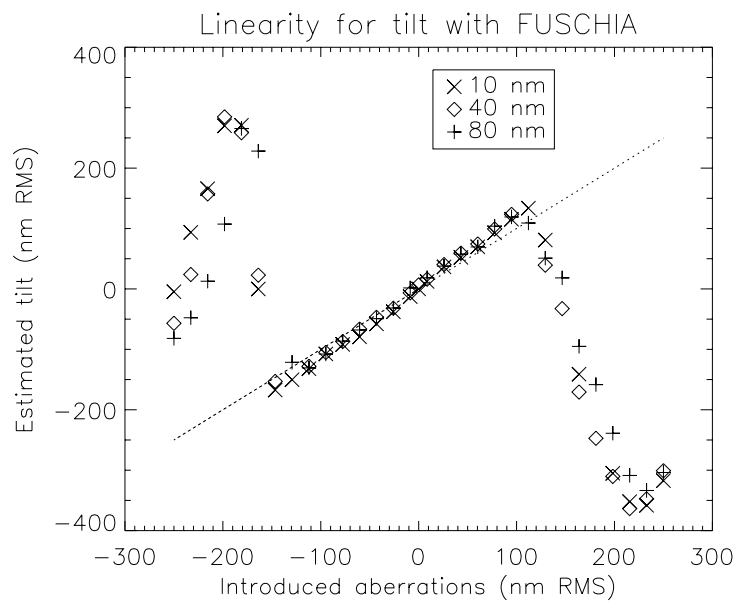

(b)

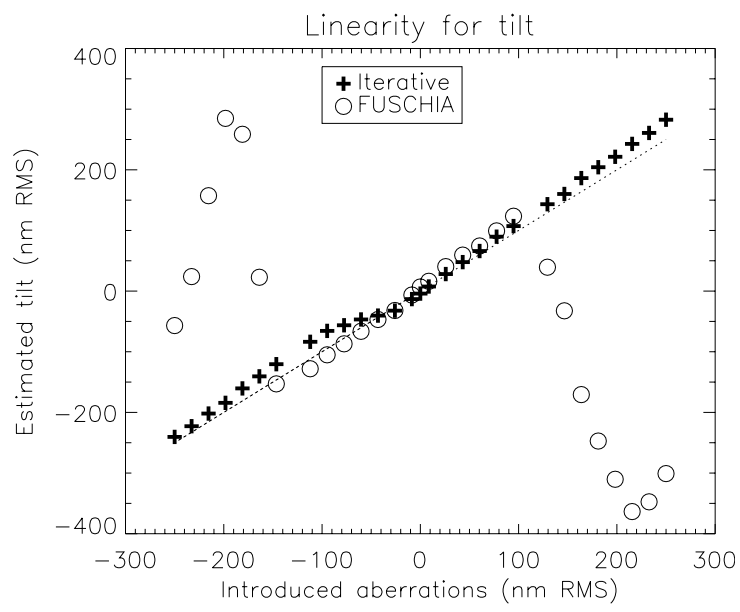

(c)

Fig. 12. Linearity of tilt estimators: (a) PSFs obtained during a tilt scan, here illustrated from $-\lambda_{c} / 3$ to $+\lambda_{c} / 7$; (b) tilt estimated with FUSCHIA for different bandwidths; (c) comparison between FUSCHIA and the iterative algorithm for the $40 \mathrm{~nm}$ bandwidth.

case. Real-time tilt correction for small aberrations (i.e., $\left.<\left|\lambda_{c} / 4\right|\right)$ is thus possible, also with large spectral band with $\Delta \lambda=\lambda_{c} / 8$.

Figure 12(c) compares the two focal-plane estimators computed on the images acquired with the $40 \mathrm{~nm}$ bandwidth. When the amplitude of the perturbation is greater than $\lambda_{c} / 4$, the iterative algorithm is initialized to the true aberrations in order to avoid local minima; in this case, tilt estimation is still possible, in contrast to estimation made with FUSCHIA: Although the three PSFs no longer superimpose, fringes created by the second ring of the tilted PSF are sufficient to correctly estimate the tip/tilt with a slope coefficient of 0.93 for the iterative estimator.

\section{Repeatability}

Figure 13 presents the standard deviation of estimated tilt with FUSCHIA and the iterative algorithm. We note that in the photon-noise regime, aberrations follow the $\gamma / N^{-0.5}$ law but with different coefficients: $\gamma=2.5$ with the iterative algorithm, whereas $\gamma$ is equal to 4.3 using FUSCHIA. This is due partially to the suboptimal use of FUSCHIA with a compact pupil: since we reject the outer parts of the OTF peaks, not all data are entirely used.

Furthermore, there are static aberrations on the reference mirror, whose values of tilt aberrations lie between $\lambda / 600$ and $\lambda / 15$, and FUSCHIA performance was shown to degrade as the value of tilt aberration increases.

Nevertheless, tip/tilt sensing at low flux is possible; with the iterative estimator, the $1.21 \mathrm{~nm}$ repeatability specified for DWARF is reached for $3.3 \times 10^{4}$ photoelectrons, which corresponds to a SNR of 11; using FUSCHIA, it is obtained for $1.6 \times 10^{5}$ photo-electrons (SNR =27). As expected, the detector noise dominates under $1.4 \times 10^{4}$ photo-electrons (i.e., $\mathrm{SNR} \leq 6$ ).

\section{CONCLUSION AND OPEN ISSUES}

The cophasing of a MAOT requires the estimation of the piston and tip/tilt on each subaperture of its pupil. Focalplane approaches, such as phase diversity, now constitute serious alternatives to pupil-plane methods. Phase diversity, however, is not very much used for cophasing, as it requires the analysis of two images and its conventional implementation is slow due to its iterative nature.

In this paper we have shown that a single focal image is sufficient for MAOT cophasing as long as the pupil is nonredundant and preferably diluted (even though slightly compact ones may still be used). Adopting a LS approach, we have derived an initial first phase-retrieval estimator. Based on a conventional iterative gradientdescent algorithm, its behavior and performance are typical of current focal-plane solutions. Then under the further assumption of small phases, we have also derived an analytical expression of the piston and tip/tilt aberrations on each subaperture and consequently a second estimator (FUSCHIA). Both algorithms were validated by simulations. Their performance in closed loop are shown to be

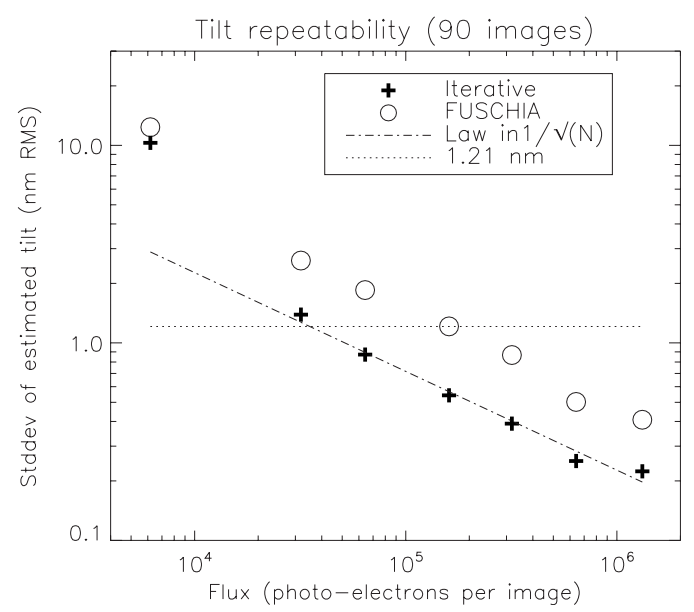

Fig. 13. Tilt repeatability estimated with FUSCHIA and the iterative algorithm. 
extremely close, and excellent overall $(<1.0 \mathrm{~nm}$ error on the piston and tilt estimates for a flux greater $10^{5}$ photoelectrons per pixel). The best use of FUSCHIA is in closed loop or in open loop for piston estimation in conjunction with the iterative estimator for tilt estimation. FUSCHIA is about 20 times faster than the iterative estimator and thus particularly suitable for real-time cophasing.

Experimental validations were also carried out on the ONERA laboratory test bench BRISE, a multipurpose test bed for cophasing sensors. Results show that using FUSCHIA or the iterative algorithm, piston can be estimated on spectral band of width $\lambda / 8$ with a subnanometric repeatability reached for a SNR of 50 . Tilt is reconstructed as long as the PSFs are superimposed; beyond this domain, FUSCHIA does not work. Between $[-\lambda / 4,+\lambda / 4]$, nanometric performance is obtained for a SNR respectively equal to 27 and 11 for FUSCHIA and the iterative algorithm. This demonstrates than an accurate real-time piston/tip/tilt correction is possible.

A rather simple cophasing sensor, composed of a focusing device, a fast focal-plane detector, and a standard computer, can thus be used to cophase a number of parallel optical beams with respect to piston and tip/tilt. Such a simple setup minimizes the number of auxiliary optics and thus the need for calibrating the differential paths. This cophasing sensor can be used near the beam combiner of a stellar interferometer, which nowadays typically includes four to eight subapertures (with adaptive optics when required). While designed for the maximum number of beams, the sensor can also operate with a smaller number of beams without any hardware change. Another application is the control of a wide-field MAOT with a pupil made of distributed circular subapertures (such as the MAOT described in Mesrine et al. [7]), using only the existing focal-plane detector and an unresolved source (star or calibration source in the object plane).

Focal-plane cophasing sensors are most probably bound to become as widely used as pupil-plane ones as they overcome their initial limitations. In particular, the methods presented in this paper can be generalized to allow the treatment of wide-spectral-bandwidth data. New developments on analytical phase diversity [29] are also a very promising alternative for future real-time cophasing systems.

\section{APPENDIX A: APPROXIMATED OTF AND PTF EXPRESSIONS FOR SMALL ABERRATIONS}

The following is a summary of several of F. Cassaing's theoretical results developed in his Ph.D. thesis [39], which have been used in the derivation of the FUSCHIA estimator. They demonstrate the existence of a modal expression of the OTF that is similar to the expression of the pupil transmission using Zernike modes.

Equation (4) shows that the OTF is the sum of $N_{T}$ $\times N_{T}$ subaperture correlations $\boldsymbol{p}_{n} \otimes \boldsymbol{p}_{n^{\prime}}$, with $\left\{n, n^{\prime}\right\}$ $\in\left[1 \ldots N_{T}\right]$. If $\boldsymbol{\phi}_{n}$ is the phase on the subaperture $n$, then

$$
\begin{aligned}
\left(\boldsymbol{p}_{n} \otimes \boldsymbol{p}_{n^{\prime}}\right)(\boldsymbol{u})= & \iint_{\boldsymbol{u}^{\prime} \in \mathbb{R}^{2}} \Pi\left(\boldsymbol{u}^{\prime}\right) \Pi\left(\boldsymbol{u}^{\prime}+\boldsymbol{u}\right) \exp j\left[\boldsymbol{\phi}_{n}\left(\boldsymbol{u}^{\prime}\right)\right. \\
& \left.-\boldsymbol{\phi}_{n^{\prime}}\left(\boldsymbol{u}^{\prime}+\boldsymbol{u}\right)\right] \mathrm{d} \boldsymbol{u}^{\prime} .
\end{aligned}
$$

Let us call $\mathcal{S}(\boldsymbol{u})$ the overlapping area between the subaperture $n$ and itself translated by $\boldsymbol{u}$. The product $\boldsymbol{\Pi}\left(\boldsymbol{u}^{\prime}\right) \Pi\left(\boldsymbol{u}^{\prime}+\boldsymbol{u}\right)$ is null outside this area. For clarity of further calculations, we will assume this product to be unity inside $\mathcal{S}(\boldsymbol{u})$ (i.e., transmissions are unity). Note then that Eq. (A1) is a sum of phasors. If the subaperture phases $\boldsymbol{\phi}_{n}$ and $\boldsymbol{\phi}_{n^{\prime}}$ are small, then this sum can be approximated by a phasor whose phase is $\Phi_{n n^{\prime}}(\boldsymbol{u})$,

$$
\begin{aligned}
& \Phi_{n n^{\prime}}(\boldsymbol{u}) \\
& \quad=\iint_{\boldsymbol{u}^{\prime} \in \mathbb{R}^{2}} \Pi\left(\boldsymbol{u}^{\prime}\right) \Pi\left(\boldsymbol{u}^{\prime}+\boldsymbol{u}\right)\left[\boldsymbol{\phi}_{n}\left(\boldsymbol{u}^{\prime}\right)-\boldsymbol{\phi}_{n^{\prime}}\left(\boldsymbol{u}^{\prime}+\boldsymbol{u}\right)\right] \mathrm{d} \boldsymbol{u}^{\prime} \\
& \quad=\iint_{\boldsymbol{u}^{\prime} \in \mathcal{S}(u)}\left[\boldsymbol{\phi}_{n}\left(\boldsymbol{u}^{\prime}\right)-\boldsymbol{\phi}_{n^{\prime}}\left(\boldsymbol{u}^{\prime}+\boldsymbol{u}\right)\right] \mathrm{d} \boldsymbol{u}^{\prime}
\end{aligned}
$$

and whose modulus is $\boldsymbol{\Lambda}(\boldsymbol{u})$ :

$$
\begin{aligned}
\boldsymbol{\Lambda}(\boldsymbol{u})=(\boldsymbol{\Pi} \otimes \boldsymbol{\Pi})(\boldsymbol{u}) & =\iint_{\boldsymbol{u}^{\prime} \in \mathbb{R}^{2}} \boldsymbol{\Pi}\left(\boldsymbol{u}^{\prime}\right) \boldsymbol{\Pi}\left(\boldsymbol{u}^{\prime}+\boldsymbol{u}\right) \mathrm{d} \boldsymbol{u}^{\prime} \\
& =\iint_{\boldsymbol{u}^{\prime} \in S(u)} 1 \mathrm{~d} \boldsymbol{u}^{\prime} .
\end{aligned}
$$

This approximation means that the OTF is considered to be the product of the unperturbed OTF $\Lambda$ by a phase function, while the effects of the aberrations on the OTF modulus are neglected:

$$
\left(\boldsymbol{p}_{n} \otimes \boldsymbol{p}_{n^{\prime}}\right)(\boldsymbol{u})=\boldsymbol{\Lambda}(\boldsymbol{u}) \exp \left[j \Phi_{n n^{\prime}}(\boldsymbol{u})\right]
$$

Expanding the phase on Zernike modes as in Eq. (3) leads to

$$
\begin{aligned}
\Phi_{n n^{\prime}}(\boldsymbol{u})= & \iint_{\boldsymbol{u}^{\prime} \in \mathcal{S}(u)} \sum_{k=1}^{3}\left[a_{k n} \boldsymbol{Z}_{k}\left(\boldsymbol{u}^{\prime}\right)-a_{k n^{\prime}} \boldsymbol{Z}_{k}\left(\boldsymbol{u}^{\prime}+\boldsymbol{u}\right)\right] \mathrm{d} \boldsymbol{u}^{\prime} \\
= & \iint_{\boldsymbol{u}^{\prime} \in \mathcal{S}(u)} \sum_{k=1}^{3}\left\{\frac{a_{k n}-a_{k n^{\prime}}}{2}\left[\boldsymbol{Z}_{k}\left(\boldsymbol{u}^{\prime}\right)+\boldsymbol{Z}_{k}\left(\boldsymbol{u}^{\prime}+\boldsymbol{u}\right)\right]\right. \\
& \left.+\frac{a_{k n}+a_{k n^{\prime}}}{2}\left[\boldsymbol{Z}_{k}\left(\boldsymbol{u}^{\prime}\right)-\boldsymbol{Z}_{k}\left(\boldsymbol{u}^{\prime}+\boldsymbol{u}\right)\right]\right\} \mathrm{d} \boldsymbol{u}^{\prime} \\
= & \sum_{k=1}^{3}\left(\alpha_{n n^{\prime}}^{k+} \mathcal{Z}_{k}^{-}+\alpha_{n n^{\prime}}^{k-}, \mathcal{Z}_{k}^{+}\right)(\boldsymbol{u}),
\end{aligned}
$$

where the following notation has been used:

$$
\alpha_{n n^{\prime}}^{k \pm}=a_{k n} \pm a_{k n^{\prime}}
$$




$$
\mathcal{Z}_{k}^{ \pm}(\boldsymbol{u})=\frac{1}{2} \iint_{\boldsymbol{u}^{\prime} \in \mathcal{S}(u)}\left[\boldsymbol{Z}_{k}\left(\boldsymbol{u}^{\prime}\right) \pm \boldsymbol{Z}_{k}\left(\boldsymbol{u}^{\prime}+\boldsymbol{u}\right)\right] \mathrm{d} \boldsymbol{u}^{\prime} .
$$

Due to the properties of Zernike polynomials, it can be shown that if the radial order of Zernike polynomial $\boldsymbol{Z}_{k}$ is even, then $\mathcal{Z}_{k}^{-}=0$; and if it is odd, $\mathcal{Z}_{k}^{+}=0$. Consequently, we will redefine $\mathcal{Z}_{k}$ as the non-null corresponding polynomials and $\alpha_{n n^{\prime}}^{k}$ as the corresponding coefficient. Thus, if $\boldsymbol{Z}_{k}$ is of even radial order,

$$
\mathcal{Z}_{k}=\mathcal{Z}_{k}^{+}, \quad \alpha_{n n^{\prime}}^{k}=\alpha_{n n^{\prime}}^{k-} .
$$

Otherwise, $\boldsymbol{Z}_{k}$ is of odd radial order:

$$
\mathcal{Z}_{k}=\mathcal{Z}_{k}^{-}, \quad \alpha_{n n^{\prime}}^{k}=\alpha_{n n^{\prime}}^{k+} .
$$

With this notation and Eq. (4), the final approximated expression of the OTF for small aberrations becomes

$$
s \approx \sum_{n=1}^{N_{T}} \sum_{n^{\prime}=1}^{N_{T}} \boldsymbol{\Lambda} \exp \left(j \sum_{k=1}^{3} \alpha_{n n^{\prime}}^{k} \mathcal{Z}_{k}\right) \star \delta_{\boldsymbol{u}_{n}-\boldsymbol{u}_{n^{\prime}}} .
$$

It can be shown that those new modes $\mathcal{Z}_{1}, \mathcal{Z}_{2}$, and $\mathcal{Z}_{3}$ are proportional to the Zernike modes $\boldsymbol{Z}_{1}, \boldsymbol{Z}_{2}$, and $\boldsymbol{Z}_{3}$ scaled to a double support. Consequently, Eq. (A12) is very similar to the expression of the transmission in the pupil plane:

$$
\boldsymbol{p}=\sum_{n=1}^{N_{T}} \boldsymbol{\Pi} \exp \left(j \sum_{k=1}^{3} a_{k n} \boldsymbol{Z}_{k}\right) \star \delta_{\boldsymbol{u}_{n}} .
$$

\section{ACKNOWLEDGMENTS}

We thank Gerard Rousset, whose ideas on phase retrieval ultimately led us to the development of this paper. We thank DGA/SPOTI for the financial support of the BRISE development and ESA for supporting DWARF. We are grateful to Stefan Hofer, Helke Schmidt, and Hans Thiele (Kayser-Threde) for manufacturing the DWARF breadboard and for fruitful discussions. Finally, we thank CNES and ONERA, who financed the first and second authors during their Ph.D. theses.

\section{REFERENCES}

1. VLTI website, http://www.eso.org/projects/vlti.

2. KECK website, http://planetquest.jpl.nasa.gov/Keck/ keck_index.html.

3. OHANA website, http://www.cfht.hawaii.edu/ lai/ ohana.html.

4. DARWIN website, http://sci.esa.int/science-e/www/area/ index.cfm?fareaid $=28$.

5. TPF website, http://planetquest.jpl.nasa.gov/TPF/ tpf_index.html.

6. JWST website, http://www.jwst.nasa.gov.

7. M. Mesrine, E. Thomas, S. Garin, P. Blanc, C. Alis, F. Cassaing, and D. Laubier, "High resolution Earth observation from Geostationary orbit by optical aperture synthesis," in Sixth International Conference on Space Optics, Proceedings of ESA/CNES ISCO 2006, A. Wilson, ed., CDROM, ESA SP-621 (2006).

8. NAOS website, http://www.eso.org/instruments/naco.

9. R. W. Gerchberg and W. O. Saxton, "A practical algorithm for the determination of phase from image and diffraction plane pictures," Optik (Stuttgart) 35, 237-246 (1972).

10. R. A. Gonsalves, "Phase retrieval from modulus data," J. Opt. Soc. Am. 66, 961-964 (1976).
11. J. G. Walker, "The phase retrieval problem: a solution based on zero location by exponential apodization," Opt. Acta 28, 735-738 (1981).

12. J. R. Fienup, "Phase retrieval algorithms: a comparison," Appl. Opt. 21, 2758-2769 (1982).

13. J. N. Cederquist, J. R. Fienup, C. C. Wackerman, S. R. Robinson, and D. Kryskowski, "Wave-front phase estimation from Fourier intensity measurements," J. Opt. Soc. Am. A 6, 1020-1026 (1989).

14. R. A. Gonsalves, "Small-phase solution to the phase retrieval problem," Opt. Lett. 26, 684-685 (2001).

15. A. Blanc, "Identification de réponse impulsionnelle et restauration d'images: apports de la diversité de phase," Ph.D. thesis (Université Paris XI Orsay, 2002).

16. J. R. Fienup, "Phase-retrieval algorithms for a complicated optical system," Appl. Opt. 32, 1737-1746 (1993).

17. J. R. Fienup, J. C. Marron, T. J. Schulz, and J. H. Seldin, "Hubble space telescope characterized by using phase retrieval algorithms," Appl. Opt. 32, 1747-1768 (1993).

18. R. A. Gonsalves, "Phase retrieval and diversity in adaptive optics," Opt. Eng. (Bellingham) 21, 829-832 (1982).

19. L. M. Mugnier, A. Blanc, and J. Idier, "Phase diversity: a technique for wave-front sensing and for diffraction-limited imaging," in Advances in Imaging and Electron Physics, P. Hawkes, ed. (Elvesier, 2006), Vol. 20, pp. 1-76.

20. A. Blanc, T. Fusco, M. Hartung, L. M. Mugnier, and G. Rousset, "Calibration of NAOS and CONICA static aberrations. Application of the phase diversity technique," Astron. Astrophys. 399, 373-383 (2003).

21. R. G. Paxman and J. R. Fienup, "Optical misalignment sensing and image reconstruction using phase diversity," J. Opt. Soc. Am. A 5, 914-923 (1988).

22. R. G. Paxman, T. J. Schultz, and J. R. Fienup, "Joint estimation of object and aberrations by using phase diversity," J. Opt. Soc. Am. A 9, 1072-1085 (1992).

23. F. Baron, "Définition et test d'un capteur de cophasage sur télescope multipupilles: application à la détection d'exoplanètes et à l'observation de la Terre," Ph.D. thesis (Ecole Doctorale d'Astronomie et d'Astrophysique d'Ile de France, 2005).

24. C. R. Vogel, T. Chan, and R. Plemmons, "Fast algorithms for phase-diversity-based blind deconvolution," Proc. SPIE 3353, 994-1005 (1998).

25. M. G. Löfdahl, A. L. Duncan, and G. B. Scharmer, "Fast phase diversity wavefront sensor for mirror control," Proc. SPIE 3353, 952-963 (1998).

26. R. L. Kendrick, D. S. Acton, and A. L. Duncan, "Phasediversity wave-front sensor for imaging systems," Appl. Opt. 33, 6533-6546 (1994).

27. G. B. Scharmer, "Object-independent fast phase-diversity," in High Resolution Solar Physics: Theory, Observations and Techniques, T. R. Rimmele, K. S. Balasubramaniam, and R. R. Radick, eds., Vol. 183 of Astron. Soc. Pacific Conf. Series (1999), pp. 330-341.

28. M. G. Löfdahl and G. B. Scharmer, "A predictor approach for closed-loop phase diversity wavefront sensing," Proc. SPIE 4013, 737-748 (2000).

29. I. Mocœur, L. M. Mugnier, and F. Cassaing, "Cophasage de télescopes multi pupilles sur scènes étendues par diversité de phase temps-réel," presented at la 26ième Colloque sur le Traitement du Signal et des Images, September 11-14, 2007, Troyes, France. Available from the authors: mugnier@onera.fr.

30. J. Idier, L. M. Mugnier, and A. Blanc, "Statistical behavior of joint least square estimation in the phase diversity context," IEEE Trans. Image Process. 14, 2107-2116 (2005).

31. C. V. M. Fridlund, "The DARWIN mission," Adv. Space Res. 34, 613-617 (2004).

32. R. J. Noll, "Zernike polynomials and atmospheric turbulence," J. Opt. Soc. Am. 66, 207-211 (1976).

33. F. Cassaing, "Optical path difference sensors," C.R. Acad. Sci. Paris, Ser. IV 21, 87-98 (2001).

34. B. Sorrente, F. Cassaing, F. Baron, C. Coudrain, B. Fleury, F. Mendez, V. Michau, L. Mugnier, G. Rousset, L. RoussetRouvière, and M.-T. Velluet, "Multiple-aperture optical 
telescopes: cophasing sensor test-bed," in Proceedings of the 5th International Conference on Space Optics, B. Warmbein, ed., Vol. SP-554 (ESA, 2004), pp. 479-484.

35. F. Cassaing, B. Sorrente, L. Mugnier, G. Rousset, V. Michau, I. Mocœur, and F. Baron, "BRISE: a multipurpose bench for cophasing sensors," Proc. SPIE 6268, 626834 (2006).

36. F. Cassaing, F. Baron, E. Schmidt, S. Hofer, L. M. Mugnier, M. Barillot, G. Rousset, T. Stuffler, and Y. Salvadé, "DARWIN Fringe Sensor (DWARF): Concept study," in Proceedings of the Conference on Towards Other Earths: $D A R W I N / T P F$ and the Search for Extrasolar Terrestrial Planets, M. Fridlund, T. Henning, and H. Lacoste, eds., Vol. SP-539 (ESA, 2003), pp. 389-392.

37. E. Schmidt, F. Cassaing, S. Hofer, M. Barillot, F. Baron, L.
M. Mugnier, G. Rousset, and T. Stuffler, "DARWIN Fringe Sensor (DWARF): breadboard development," in Proceedings of the Conference on Towards Other Earths: DARWIN/TPF and the Search for Extrasolar Terrestrial Planets, M. Fridlund, T. Henning, and H. Lacoste, eds., Vol. SP-539, (ESA, 2003), pp. 575-577.

38. J. H. Seldin, R. G. Paxman, V. G. Zarifis, L. Benson, and R. E. Stone, "Closed-loop wavefront sensing for a sparseaperture, phased-array telescope using broadband phase diversity," Proc. SPIE 4091, 48-63 (2000).

39. F. Cassaing, "Analyse d'un instrument à synthèse d'ouverture optique: méthodes de cophasage et imagerie à haute résolution angulaire," Ph.D. thesis (Université Paris XI Orsay, 1997). 